\title{
Focused ultrasound excites neurons via mechanosensitive calcium accumulation and ion channel amplification
}

\author{
Sangjin Yoo ${ }^{1}$, David R. Mittelstein ${ }^{2}$, Robert Hurt ${ }^{3}$, Jerome Lacroix ${ }^{1,4}$, Mikhail G. Shapiro ${ }^{1 *}$ \\ ${ }^{1}$ Division of Chemistry and Chemical Engineering, California Institute of Technology, Pasadena, CA 91125, USA \\ ${ }^{2}$ Division of Engineering and Applied Science, California Institute of Technology, Pasadena, CA 91125, USA \\ ${ }^{3}$ Division of Biology and Biological Engineering, California Institute of Technology, Pasadena, CA 91125, USA \\ ${ }^{4}$ Graduate College of Biomedical Sciences, Western University of Health Sciences, 309 E. Second St, Pomona, CA 91766, USA \\ *Correspondence should be addressed to M.G.S. (mikhail@caltech.edu)
}

\begin{abstract}
Ultrasonic neuromodulation has the unique potential to provide non-invasive control of neural activity in deep brain regions with high spatial precision and without chemical or genetic modification. However, the biomolecular and cellular mechanisms by which focused ultrasound excites mammalian neurons have remained unclear, posing significant challenges for the use of this technology in research and potential clinical applications. Here, we show that focused ultrasound excites neurons through a primarily mechanical mechanism mediated by specific calcium-selective mechanosensitive ion channels. The activation of these channels results in a gradual build-up of calcium, which is amplified by calcium- and voltage-gated channels, generating a burst firing response. Cavitation, temperature changes, large-scale deformation, and synaptic transmission are not required for this excitation to occur. Pharmacological and genetic inhibition of specific ion channels leads to reduced responses to ultrasound, while over-expressing these channels results in stronger ultrasonic stimulation. These findings provide a critical missing explanation for the effect of ultrasound on neurons and facilitate the further development of ultrasonic neuromodulation and sonogenetics as unique tools for neuroscience research.
\end{abstract}

\section{INTRODUCTION}

Non-invasive neuromodulation technologies play a critical role in basic neuroscience research and the development of therapies for neurological and psychiatric disease. However, established non-invasive techniques such as transcranial magnetic stimulation (TMS) and transcranial direct current stimulation (tDCS) suffer from limited spatial targeting and penetration depth(Wagner et al., 2007). In contrast, focused ultrasound (FUS) has the potential to modulate neural activity in deep-brain regions with millimeter spatial precision based on the penetrance of sound waves in bone and soft tissue. Recently, transcranial FUS in the frequency range of $0.25-1$ $\mathrm{MHz}$ and intensity of $1-100 \mathrm{~W} / \mathrm{cm}^{2}$ (I I $_{\text {SPA }}$ ) has been shown to elicit neural and behavioral responses in small (Kim et al., 2012; King et al., 2013; Sharabi et al., 2018; Tufail et al., 2010; Ye et al., 2016; Younan et al., 2013) and large (Dallapiazza et al., 2018; Deffieux et al., 2013; Folloni et al., 2019; Lee et al., 2016b; Verhagen et al., 2019; Wattiez et al., 2017; Yoo et al., 2011 ) model animals and humans (Lee et al., 2015; Lee et al., 2016a; Legon et al., 2018a; Legon et al., 2014; Leo Ai, 2018) without genetic or chemical alterations or deleterious side effects, even with chronic stimulation (Bystritsky et al., 2011). These studies have driven widespread interest in the development of FUS as a research tool in neuroscience and a strategy for disease treatment (Naor et al., 2016).

Despite the intense interest in this technology, the underlying cellular and molecular mechanisms of ultrasonic neuromodulation are largely unknown. The study of these mechanisms is made challenging as ultrasound produces multiple physical effects, including mechanical force, heating and cavitation (Krasovitski et al., 2011; O'Brien, 2007; Plaksin et al., 2016; Plaksin et al., 2014; Tyler, 2011, 2012; Tyler et al., 2008). The role of these physical processes in neuromodulation and their transduction to molecular signals in neurons have not been elucidated. Moreover, recent findings of off-target auditory effects of FUS in small animals make it challenging to study potential mechanisms in the in vivo context (Guo et al., 2018; Sato et al., 2018).

Here, we describe a comprehensive study of the molecular and cellular mechanisms of ultrasonic neuromodulation in primary cortical neurons. Using stimulation and readout methods consistent with the acoustic conditions expected in vivo, we first narrow down the biophysical basis by which ultrasound excites neurons, observing no involvement of temperature elevation, cavitation or large-scale deformation. Then, we uncover a signaling pathway whereby the mechanical effects of ultrasound cause calcium influx through specific endogenous mechanosensitive ion channels. We find that this triggers signal amplification by calcium-gated sodium channels, and ultimately results in robust spiking activity. This pathway functions internally within neurons and does not require synaptic transmission. The overexpression of specific mechano-sensitive and amplifier channels identified in our biophysical experiments significantly enhances ultrasound response magnitude and kinetics. These results provide comprehensive mechanistic insights into the excitatory action of ultrasound on mammalian neurons, with important implications for the development of ultrasonic neuromodulation and sonogenetics.

\section{RESULTS}

\section{Focused ultrasound robustly activates cortical neurons}

To study neuronal responses to focused ultrasound under acoustic conditions matching soft tissue, we cultured primary 
bioRxiv preprint doi: https://doi.org/10.1101/2020.05.19.101196; this version posted May 22, 2020. The copyright holder for this preprint (which was not certified by peer review) is the author/funder. All rights reserved. No reuse allowed without permission.

a

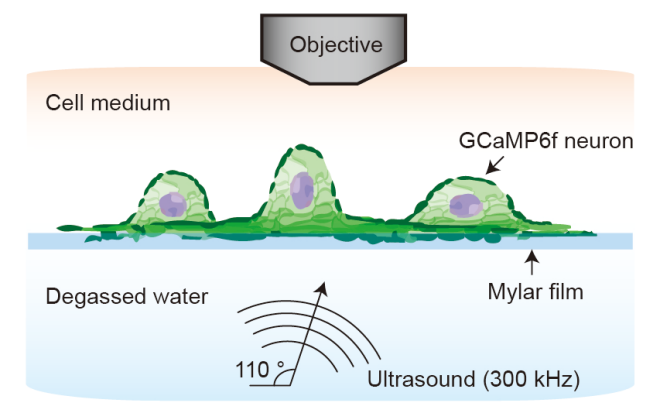

b

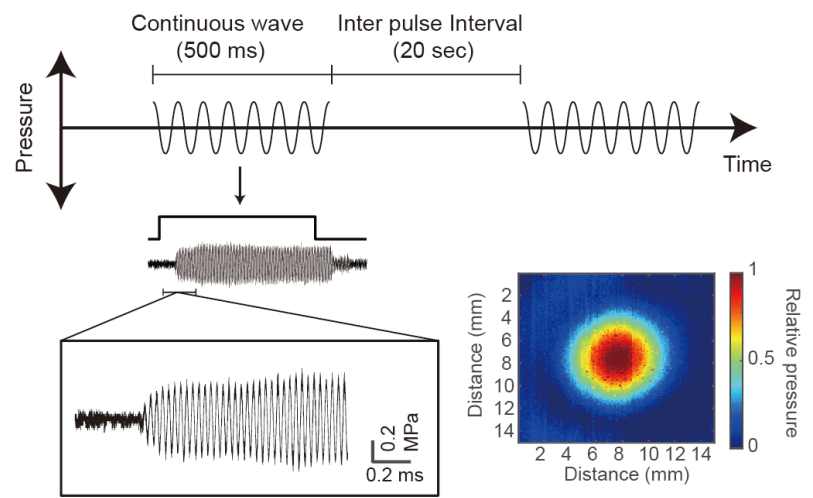

c
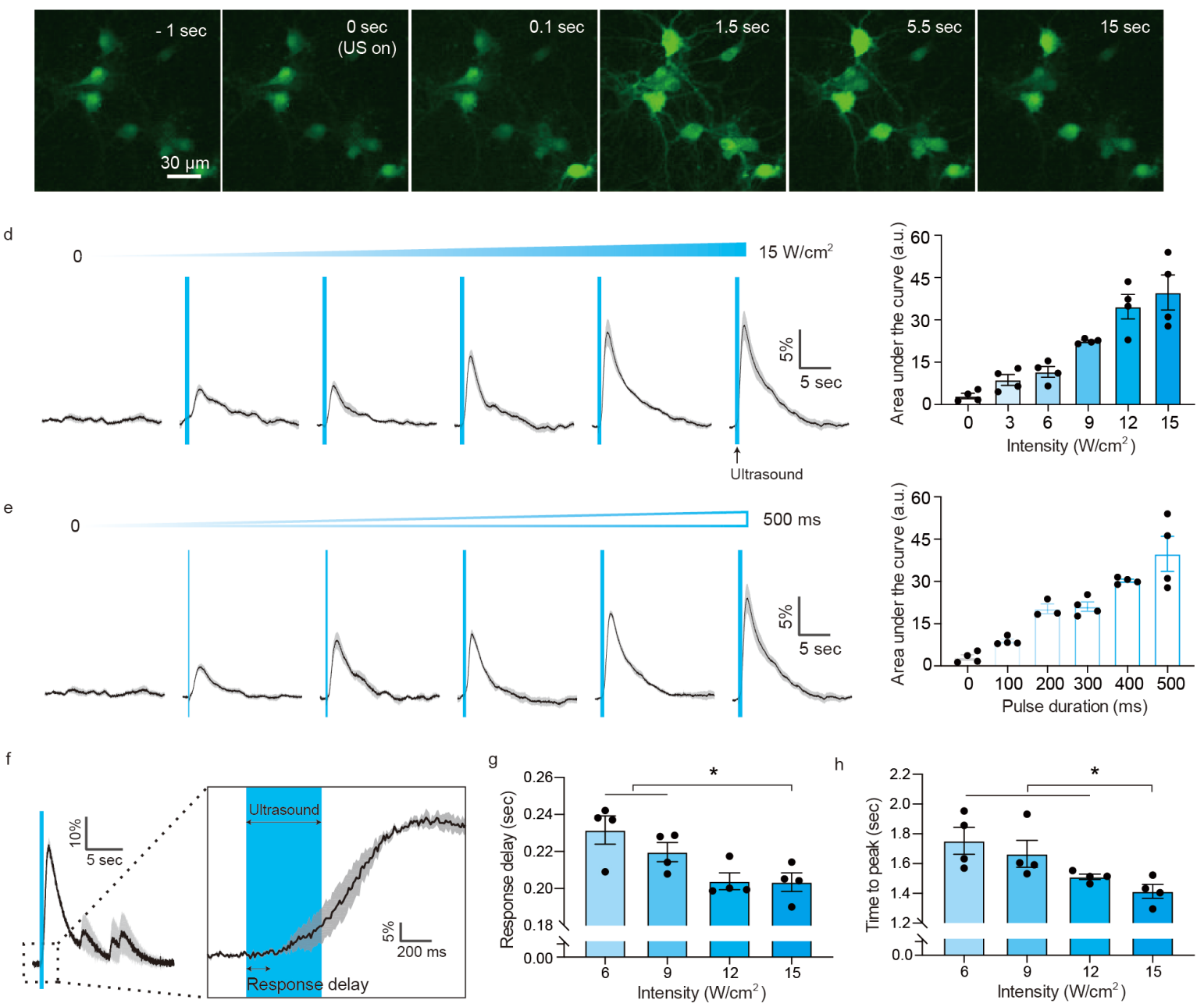

Fig. 1 | Gultured cortical neurons are excited by focused ultrasound stimulation. (a) Illustration of the focused ultrasound stimulation setup. Angled ultrasound waves are delivered to GCaMP6f-expressing neurons cultured on an acoustically transparent a mylar film, while the neural calcium response is recorded by epifluorescence imaging. (b) Schematic of the acoustic waveform applied to neurons and representative focal pressure waveform measured by a hydrophone. The colormap shows the spatial profile of the acoustic pressure at the ultrasound focus, with a full-width at half-maximal diameter $5.2 \mathrm{~mm}$. (c) Representative time lapse images of GCaMP6f fluorescence before, during and after ultrasound stimulation $\left(15 \mathrm{~W} / \mathrm{cm}^{2}, 500 \mathrm{~ms}\right.$ pulse duration). (d) Calcium responses and quantification of neural response as function of ultrasound intensity $(n=4$ dishes each, one-way ANOVA $p<0.0001)$ and (e) pulse duration $(n=4$ dishes each, one-way ANOVA $\mathrm{p}>0.0001$ ). (f) A representative single cell response to ultrasound. (g) Quantification of response onset time (one-way ANOVA p=0.0117, Tukey's post comparison) and (h) time to peak (one-way ANOVA $\mathrm{p}=0.0190$, Tukey's post comparison). Mean trace in solid and SEM is shaded.

murine cortical neurons on an acoustically transparent mylar film while optically recording their calcium and voltage responses to ultrasound using genetically encoded fluorescent indicators $($ Fig. 1a). The neurons were placed at the top of a water tank, with a focused ultrasound transducer submerged in degassed water below them and angled to reduce standing 
wave formation. The $5 \mathrm{~mm}$ focal diameter of the transducer (Fig. 1b) delivered ultrasound uniformly to neurons throughout our field of view. We used a frequency of $300 \mathrm{kHz}$, within the range utilized in recent studies in a variety of organisms (Deffieux et al., 2013; Folloni et al., 2019; Lee et al., 2015; Lee et al., 2016a; Legon et al., 2018a; Legon et al., 2018b; Legon et al., 2014; Leo Ai, 2018; Wattiez et al., 2017), and continuous-wave stimulation, which was found to be as effective as pulsed ultrasound (King et al., 2013). The interpulse interval was fixed at $20 \mathrm{sec}$ to allow a return to baseline.

To establish the ultrasound pulse parameters under which neurons in culture respond to ultrasound, we stimulated the cells across a range of pulse intensities $\left(0-15 \mathrm{~W} / \mathrm{cm}^{2}\right)$ and pulse durations $(0-500 \mathrm{~ms})$ while imaging calcium responses with virally transfected GCaMP6f (Fig. 1c, Supplementary Video 1). The neurons showed robust responses, with amplitudes increasing monotonically with intensity and pulse duration (Fig. 1, d-e). The calcium response was not immediate, but had a delay of approximately $200 \mathrm{~ms}$ after the onset of stimulation (Fig. 1f). This onset delay is not explained by the rise time of GCaMP6f fluorescence (time to peak $\sim 45$ $\mathrm{ms})$ (Chen et al., 2013), and therefore reflects the kinetics of the neurons' response to FUS. Both this onset delay and the maximum response time $(\sim 1.7 \mathrm{sec})$, were reduced significantly by increasing ultrasound intensity (Fig. 1g-h). Based on these results, we set our subsequent stimulation parameters to 15 $\mathrm{W} / \mathrm{cm}^{2}$ and $500 \mathrm{~ms}$, which are similar to those used in large animal and human studies (Deffieux et al., 2013; Folloni et al., 2019; Lee et al., 2015; Lee et al., 2016a; Legon et al., 2018a; Legon et al., 2018b; Legon et al., 2014; Leo Ai, 2018; Wattiez et al., 2017). To ensure that these ultrasound parameters were not damaging to cells, we looked for and found neither sustained calcium accumulation nor irreversible membrane perforation after repetitive stimuli (Supplementary Fig. 1).

To determine whether the observed responses to FUS were specific to the adherent 2D culture format, we also applied ultrasound to neurons in 3D collagen culture. We found that neurons in this format also showed reliable calcium signals in response to stimulation (Supplementary Fig. 2). In addition, to determine the extent to which standing waves, which are nearly impossible to completely eliminate in a FUS setup in vitro and in vivo (O'Reilly et al., 2010; Tang and Clement, 2010), play a role in the observed excitation, we applied FUS to neurons with a chirped waveform, which ameliorates the pressure gradients induced by standing waves. We found the calcium signal unaffected in terms of response amplitude (Supplementary Fig. 3).

\section{Ultrasound excites neurons via mechanical force}

Focused ultrasound is capable of producing multiple physical phenomena in tissue, including elevating temperature, inducing bubble formation and cavitation, and applying mechanical force $(\mathbf{F i g}$. 2a), each of which could potentially lead to neuronal excitation (Ibsen et al., 2015; Krasovitski et al., 2011; Plaksin et al., 2016; Plaksin et al., 2014; Shapiro et al., 2012). To determine which of these phenomena are involved in stimulating cortical neurons, we first measured changes in temperature during FUS application. A fiber optic thermometer positioned adjacent to the neurons recorded temperature changes of $0.005 \pm 0.003{ }^{\circ} \mathrm{C}$ in response to our optimized ultrasound parameters (Fig. 2b), and changes below $0.02{ }^{\circ} \mathrm{C}$ at all parameters tested using a $300 \mathrm{kHz}$ transducer (Supplementary Fig. 4a). The absence of a major temperature increase was corroborated by co-expressing the fluorescent protein mCherry (Guo et al., 2012) as a temperature indicator (Supplementary Fig. 4b), and showing that the mCherry fluorescence stayed constant while neurons responded to ultrasound (Fig. 2c). These results confirm that temperature does not play a role in ultrasonic neuromodulation in this parameter range, as predicted by numerical estimates (Constans et al., 2018; Lee et al., 2018).

Among the potential non-thermal effects of ultrasound, bubble formation and cavitation have been hypothesized as a mechanism for ultrasonic neuromodulation due to the observation of enhanced responses at lower frequencies (Tyler, 2011). To assess the relevance of this phenomenon in cultured neurons, we compared their responses to ultrasound in atmospherically gassed and degassed cellular media, with the latter condition disfavoring cavitation. No significant differences were observed (Fig. 2d). In addition, we looked for bubbles directly using an ultra-high-speed camera $(5 \mathrm{MHz}$ frame rate), and saw no bubbles formed in the vicinity of neurons during FUS application (Fig. 2e, Supplementary Fig. 4c). These results are consistent with our mechanical index $(\mathrm{MI}=0.9)$ being below the typical cavitation threshold in soft tissue (MI > 1.9) (Nightingale et al., 2015). Although the optical resolution of our setup $(\sim 532 \mathrm{~nm})$ was insufficient to directly visualize the formation of the nanoscale bubbles hypothesized by some theoretical studies to form inside the membrane bilayer (Krasovitski et al., 2011; Plaksin et al., 2016; Plaksin et al., 2014), the gross appearance of the membrane remained unchanged over the ultrasound cycle $(\mathbf{F i g}$. 2f). This suggests that there were no major changes in refractive index, as might be expected if a large fraction of the membrane surface undergoes cavitation, as required by the intramembrane cavitation theory (Krasovitski et al., 2011; Plaksin et al., 2016; Plaksin et al., 2014). The voltage implications of the theory are further examined in the next section of the manuscript.

Having ruled out temperature changes and cavitation, we next focused on direct mechanical forces. Given the similar acoustic impedance of neurons and surrounding media, the acoustic radiation force on the neurons due to FUS is expected to be weak, with any resulting deformations expected to be below our optical detection limit. Indeed, under high-speed imaging we observed no significant cell deformation either during each wave cycle (Fig. 2g, Supplementary Fig. 4d) or over the longer course of the ultrasound pulse (Fig. 2f). However, as an indirect test of the involvement of mechanical deformation in the neuromodulation response, we altered the mechanical properties of the neurons by depolymerizing their actin cytoskeleton, which plays a critical role in establishing the elastic modulus of the cytoplasm and cellular cortex. When we depolymerized actin using cytochalasin D (Cooper, 1987), at concentrations that did not affect spontaneous excitability or viability of the neurons $($ Fig. 2h, Supplementary Fig. 4e) (Cooper, 1987; Wang et al., 2003), we observed a significant reduction in the amplitude of the evoked calcium response (Fig. 
a

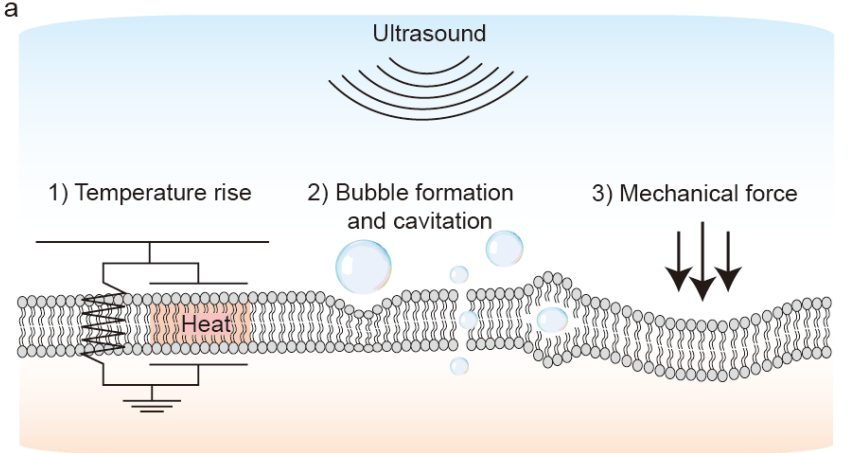

b

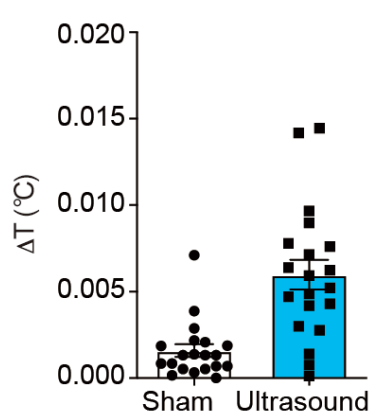

c

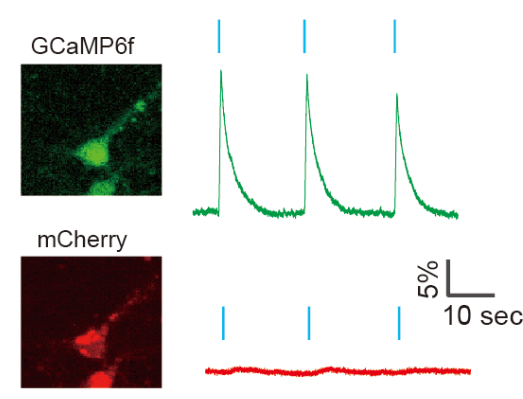

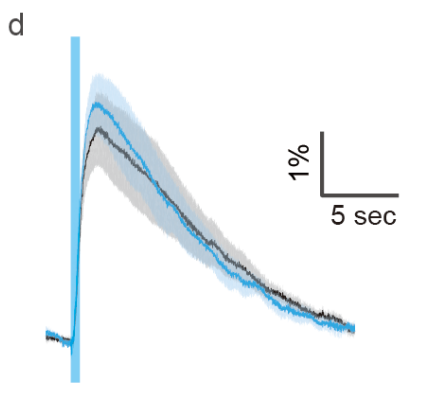
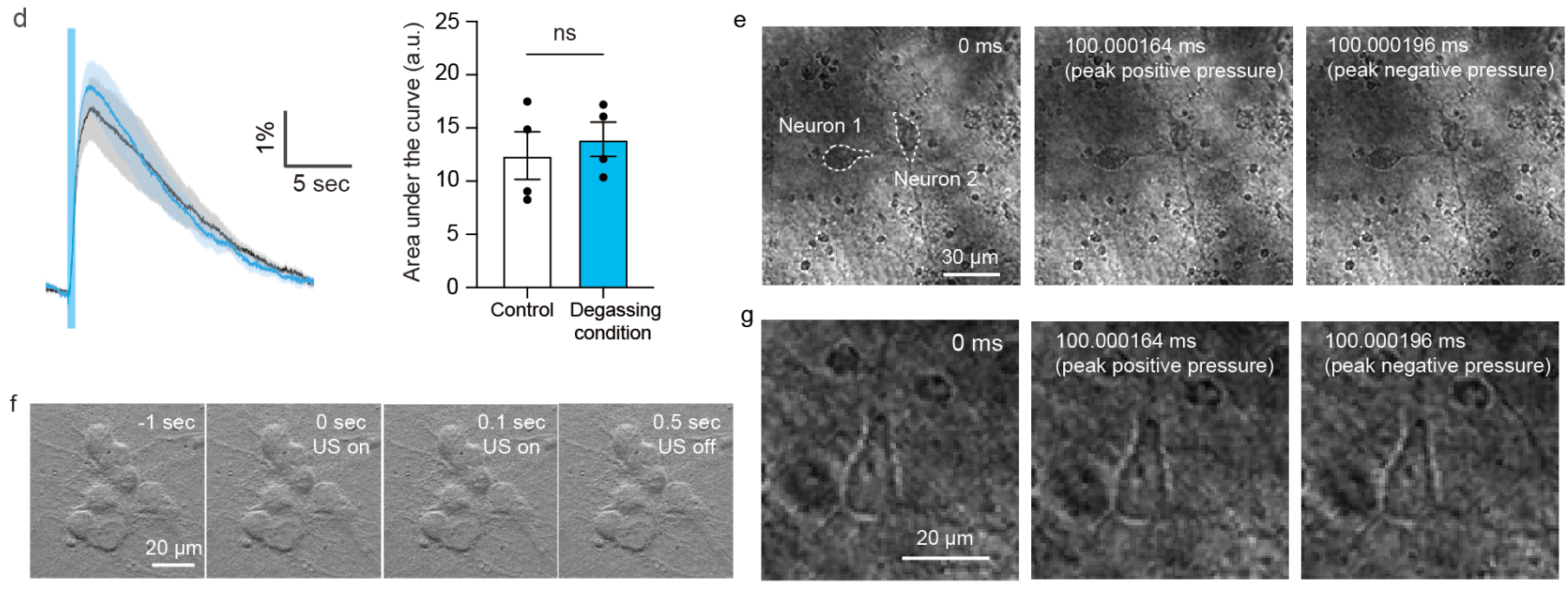

$\mathrm{h}$
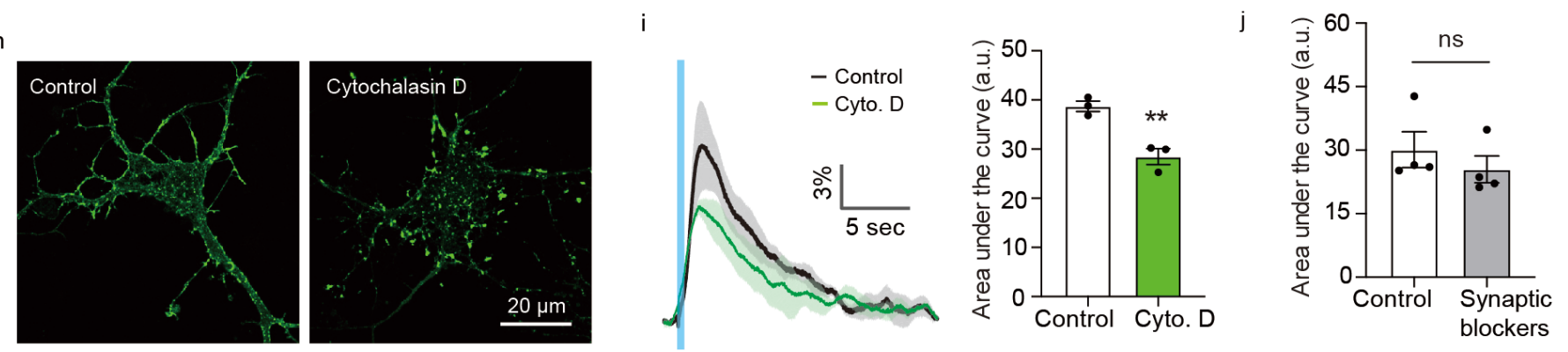

Fig. 2 | Ultrasound excites neurons through direct mechanical effects. (a) Illustration of the potential biophysical effects of ultrasound. (b) Temperature increase measured using an optic hydrophone thermometer positioned near the neurons during ultrasound stimulation ( $\mathrm{n}=20$, $15 \mathrm{~W} / \mathrm{cm}^{2}, 500 \mathrm{~ms}$ pulse duration with 2 sec inter-pulse interval). (c) Fluorescence images of a neuron co-expressing GCaMP6f (green) and mCherry (red) and changes in their respective fluorescence in response to ultrasound stimulation. (d) Calcium responses to ultrasound in freshly degassed media ( $\mathrm{n}=4$ dishes each, unpaired T-test, $\mathrm{p}=0.6033)$. (e) Ultra-high-speed imaging (5 Mfps) of neurons and surrounding media during ultrasound stimulation. Image recording was started $100 \mathrm{~ms}$ after the onset of ultrasound. (f) Ultra-high-speed imaging of a single neuron during ultrasound stimulation at higher magnification. (g) Bright field imaging of neurons over the full time course of the ultrasound stimulation. (h) Images of the F-actin label Alexa-Fluor 488 phalloidin before and after treatment with cytochalasin D. (i) Calcium responses before and after cytochalasin D treatment, and quantification of area under the curve $(\mathrm{n}=3$ dishes, unpaired T-test, $\mathrm{p}=0.0061)$. ( $\mathrm{j}) \mathrm{Quantification}$ of area under the after applying the synaptic blockers AP5 and CNQX ( $1 \mu \mathrm{M}$ each, $\mathrm{n}=4$ dishes, Paired T test, $\mathrm{p}=0.4128)$. Mean trace in solid and SEM is shaded.

2i). This suggests that mechanical stress is involved in ultrasonic neuromodulation, albeit in a manner not resulting in, or requiring, micron-scale deformation of the cell.

As a final question before delving into molecular mechanisms, we asked whether the neuronal response to ultrasound was cellautonomous or required synaptic connections with excitatory neurons (Tyler et al., 2008) or astrocytes (Oh et al., 2020). After treating the neurons with the postsynaptic blockers AP5 and
CNQX, we found that the neuronal response to ultrasound was unaffected $(\mathbf{F i g}$. 2j), suggesting that each neuron responds to ultrasound on its own.

\section{Ultrasound stimulation triggers calcium entry across the plasma membrane}

To determine the molecular basis of the neuronal response to ultrasound, we first examined which ions enter the cell during 

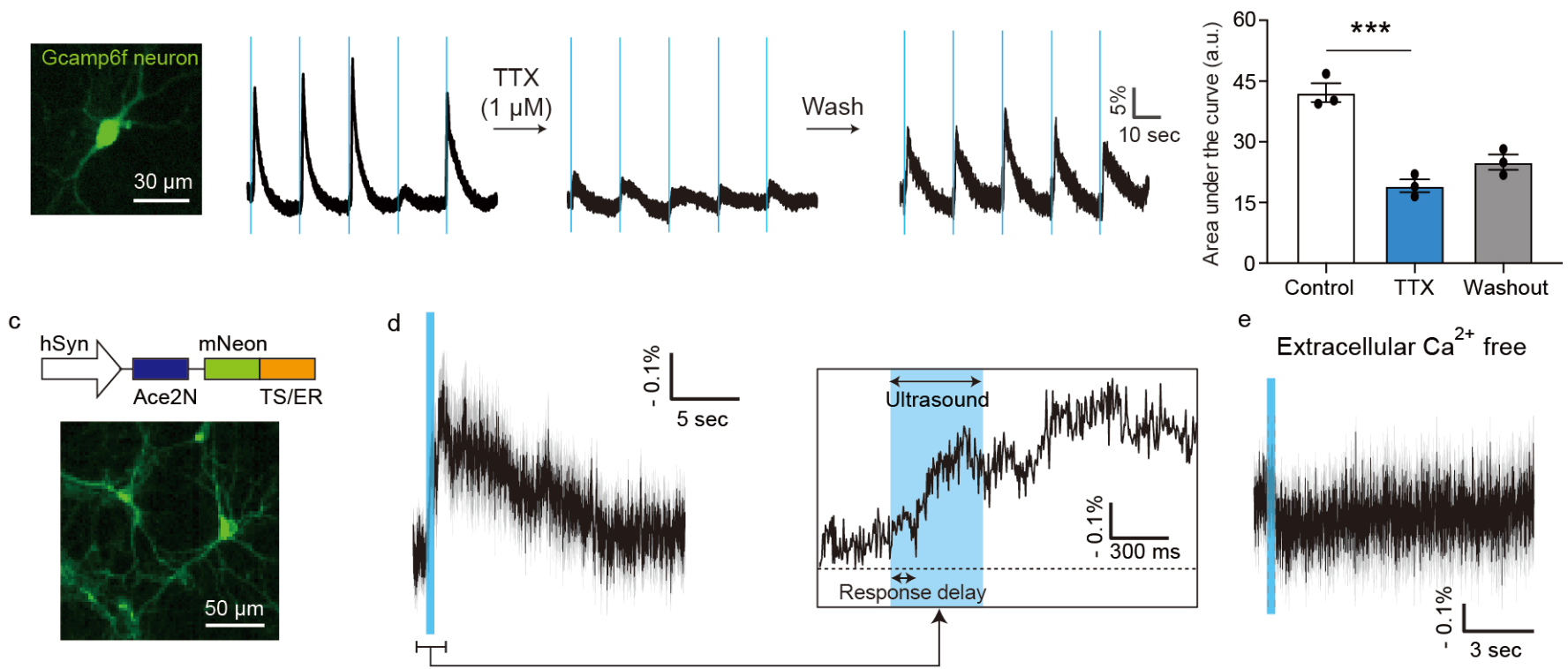

Fig.3 | Ultrasound response is mediated by the entry of extracellular calcium. (a) Calcium responses from a single neuron during ultrasound stimulation before, during and after treatment with the sodium channels blocker tetrodotoxin (TTX). (b) Quantification of area under the curve before, during and after TTX treatment $(n=3$ dishes, one-way ANOVA $\mathrm{p}=0.0004$, Tukey's post comparison (control vs TTX)). (c) Diagram of the Ace2N voltage indicator genetic construct and representative fluorescence image of neurons transfected with this construct. (d) Voltage responses to ultrasound. ( $n=4$ dishes). (e) Voltage responses of neurons in calcium-free media $(n=2$ dishes). Mean trace in solid and SEM is shaded.

FUS stimulation. Since neurons contain voltage-gated $\mathrm{Ca}^{2+}$ channels, it is not possible to determine from GCaMP responses alone whether $\mathrm{Ca}^{2+}$ enters the cell directly as a result of ultrasound or due to action potential firing. However, consistent with previous results in slices (Tyler et al., 2008), blocking voltage-gated sodium channels with TTX only partially reduced the magnitude of the ultrasound response (Fig. 3, a-b). This suggests that calcium enters the cell directly as a result of ultrasound application, in addition to its entry following depolarization. To confirm the role of $\mathrm{Ca}^{2+}$ as a primary initiator of the response to FUS, we imaged transmembrane voltage using the genetically encoded voltage indicator Ace2N (Fig. 3c, Supplementary Fig. 5a). In the normal, calcium-containing media, FUS application elicited depolarization, with a response onset similar to that observed with GCaMP6f (Fig. 3d). In contrast, in calcium-free media this voltage response to ultrasound was completely eliminated (Fig. 3e), while the cells retained their ability to respond to other stimuli (Supplementary Fig. 5b). These results demonstrate that extracellular $\mathrm{Ca}^{2+}$ is the essential ionic initiator of ultrasonic neuromodulation. Intracellular calcium release from the endoplasmic reticulum does not play a major additional role (Supplementary Fig. 6).

Voltage imaging also provided an additional method to test the intramembrane cavitation theory, which hypothesizes that bubble formation leads to rapidly oscillating hyperpolarizing currents, resulting in action potential generation through a charge accumulation mechanism (Krasovitski et al., 2011; Plaksin et al., 2016; Plaksin et al., 2014). Although the kinetics of our voltage sensor are not fast enough to capture membrane potential oscillation at the ultrasound frequency, we would expect it to pick up time-averaged hyperpolarization during ultrasound application. However, no such hyperpolarization was observed (Fig. 3d), and this result was corroborated in spiking HEK cells as a generic excitable membrane model (Supplementary Fig. 5, c-d).

\section{Ultrasound stimulation activates specific mechanosensitive ion channels}

Having established that ultrasound excites neurons via mechanical force resulting in the entry of extracellular calcium, we hypothesized that this response involves the activation of endogenous mechanosensitive ion channels (Christensen and Corey, 2007; Kubanek et al., 2018; Lee et al., 2015; Tyler, 2011). Cortical neurons have been shown to express multiple channels with reported mechanosensitivity, including TRPV1, TRPV2, TRPV4, Piezol, TRPG1, TRPM7 and the TRPP1/2 complex (Christensen and Corey, 2007; Ranade et al., 2015). Mechanosensitive ionic currents can also be mediated indirectly by G-protein coupled receptors (GPCRs) (Xu et al., 2018). To determine which channels are involved in ultrasonic neuromodulation, we first blocked subsets of candidate mechanosensitive receptors using pharmacological blockers, then used CRISPR/Cas9 knockdown to further delineate the roles of specific proteins $(\mathbf{F i g} . \mathbf{4 a})$.

We started by treating the neurons with gadolinium(III), a global mechanosensitive channel inhibitor which acts by modifying the deformability of the lipid bilayer (Cheng et al., 1999). The dose of $\mathrm{Gd}^{3+}$ was carefully chosen to avoid blocking non-mechanosensitive channels or otherwise altering cell excitability (Hamill and McBride, 1996) (Supplementary Fig. 7a). In the presence of $\mathrm{Gd}^{3+}$, the amplitude of the evoked 


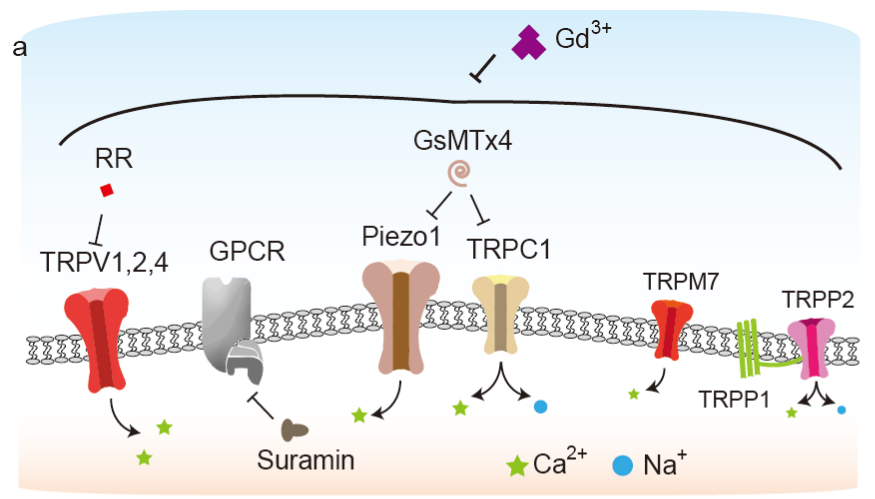

b
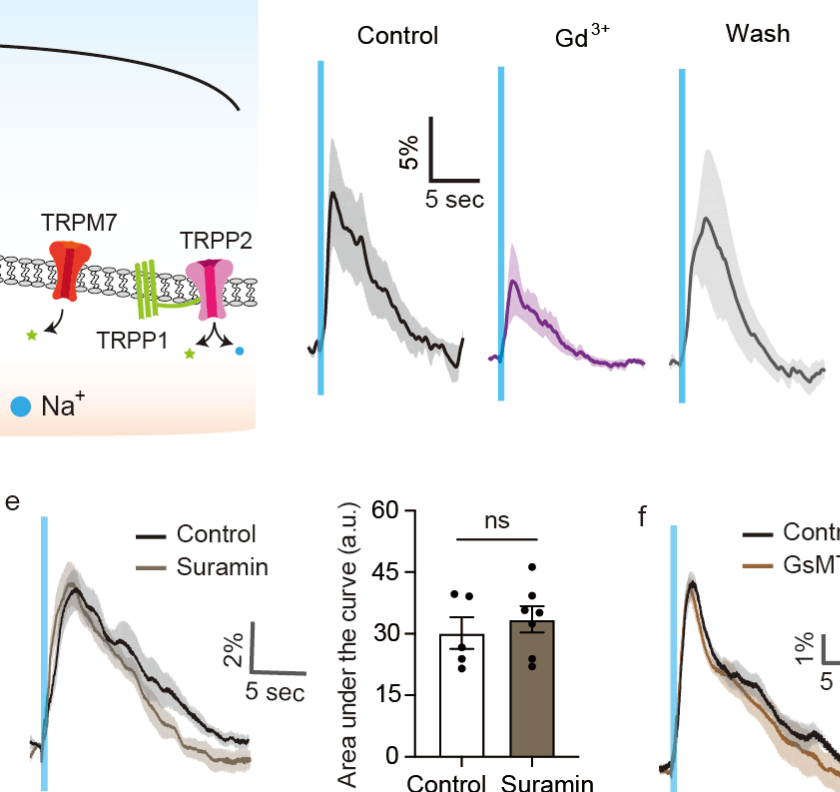

C
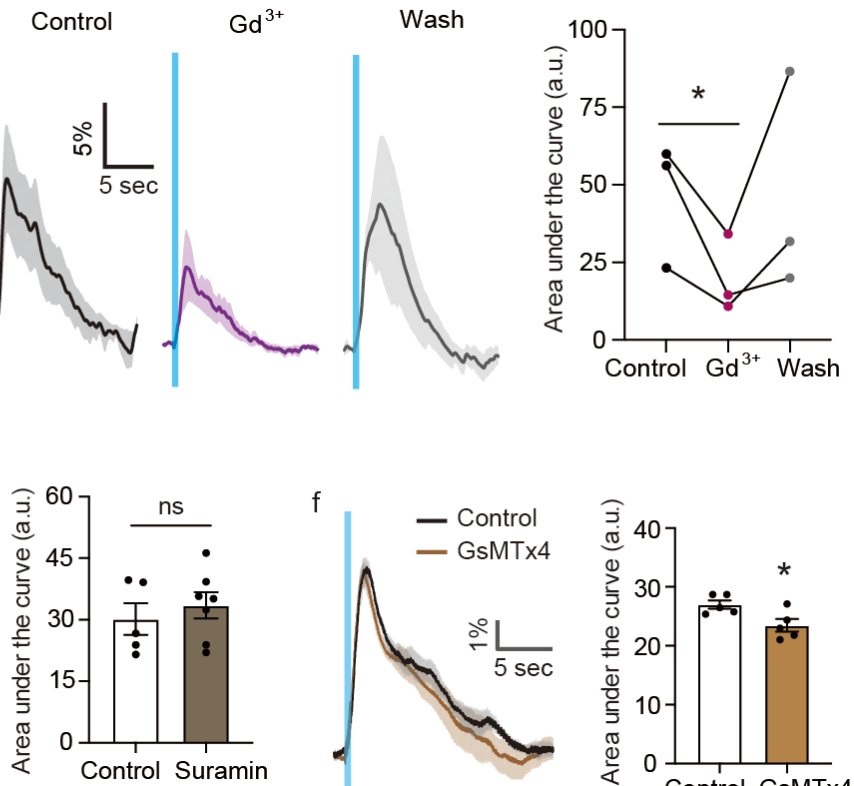

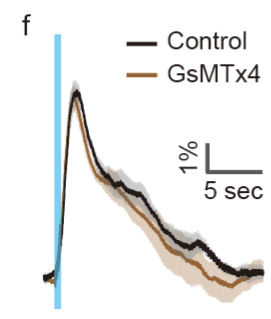

d

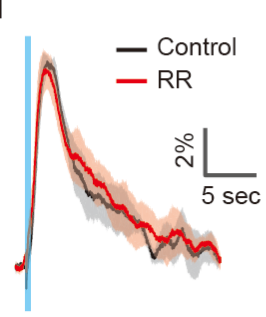

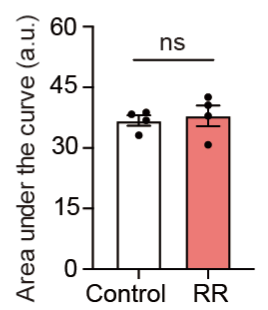

Fig.4 | Pharmacological inhibition of mechanosensitive receptors. (a) Schematic of neuronal mechanosensitive receptors and strategies to block them. Gadolinium $\left(\mathrm{Gd}^{3+}, 20 \mu \mathrm{M}\right)$ was used to block the mechanosensitive channels nonspecifically. Pores of TRPV1, 2 and 4 channels were blocked by ruthenium red $(\mathrm{RR}, 1 \mu \mathrm{M})$. Activation of GPCRs was inhibited by suramin $(60 \mu \mathrm{M})$. Gating of Piezol and TRPC1 channels was inhibited by GsMTx4 $(10 \mu \mathrm{M})$. (b) Calcium responses before, during and after treatment the Gd ${ }^{3+}$. (c) Average calcium response under each condition $(\mathrm{n}=3$ dishes, Paired T-test, $\mathrm{p}=0.0884)$. (d) Calcium responses before and after treatment with $\mathrm{RR}(\mathrm{n}=3$ dishes, unpaired T-test, $\mathrm{p}=0.6930$ ). (e) Calcium responses before and after treatment with suramin $(\mathrm{n}=5$ dishes for control, and 7 dishes for suramin, unpaired $\mathrm{T}$ test, $\mathrm{p}=0.5159$ ). (f) Calcium responses before and after treatment with GsMTx4 ( $\mathrm{n}=5$ dishes each, unpaired T test, $\mathrm{p}=0.0245)$. Mean trace in solid and SEM is shaded.

responses was significantly reduced, decreasing by $60 \%$ (Fig. $\mathbf{4}, \mathbf{b}-\mathbf{c})$. This confirmed that mechanosensitive channels are involved in ultrasound transduction.

Next, we used selective chemical blockers to inhibit distinct mechanosensitive channels, carefully titrating each drug to avoid non-specific excitability reduction or cytotoxicity (Supplementary Fig. 7, b-e). First, we used ruthenium red $\left(\mathrm{IC}_{50} \sim 500 \mathrm{nM}\right.$, used at $\left.1 \mu \mathrm{M}\right)$ to block TRPV1, TRPV2 and TRPV4 channels (Vriens et al., 2009). The resulting neural responses were not significantly different from controls (Fig. 4d), suggesting that these channels are not involved. Next, we used suramin, which blocks GPCR signaling by inhibiting the release of GDP from the $\mathrm{G}$ alpha subunit (Freissmuth et al., 1996) $\left(\mathrm{IC}_{50} \sim 200 \mathrm{nM}\right.$, used at $\left.60 \mu \mathrm{M}\right)$. Neurons treated with this compound showed no significant change in their response to ultrasound compared to controls $(\mathbf{F i g}$. 4e), suggesting that GPCRs are not involved. We then tested the involvement of the Piezol and TRPCl channels using the peptide inhibitor GsMTx4 $\left(\mathrm{IC}_{50} \sim 5 \mu \mathrm{M}\right.$, used $10 \mu \mathrm{M}$ in this study), which inserts into the stressed membrane and distorts membrane tension near the channels (Gnanasambandam et al., 2017; Spassova et al., 2006). Neurons treated with GsMTx4 $\left(\mathrm{IC}_{50} \sim 5 \mu \mathrm{M}\right.$, used at $10 \mu \mathrm{M}$ ), showed a modest but significant reduction in the magnitude of their response to ultrasound (Fig. 4f). This indicates the partial involvement of Piezol and/or TPRC1 channels in ultrasonic neuromodulation.

Because selective pharmacological inhibition was not available for all the candidate channels, we also used CRISPR/Cas9 to knock down several of channels $(\mathbf{F i g}$. 5a). For each channel, sgRNA sequences were designed using the CRISPRko tool (Doench et al., 2016) to maximize targeted Cas9 activity and minimize off-target effects. The designed sgRNA was inserted into an all-in-one vector, containing a single sgRNA expression cassette and a Cas9 nuclease expression cassette, and delivered to neurons via lentivirus (Sanjana et al., 2014) (Fig. 5b). When sgRNAs were used to target TRMP7, TRPP1, TRPP2, Piezo1 and TRPC1, they produced editing efficiencies of 20.0 to $39.6 \%$, as quantified by decomposing the target sequence traces (Supplementary Fig. 8a) (Brinkman et al., 2014). The effect of the partial knockdown of each channel was measured by plotting the average calcium response and quantifying the change in the magnitude of the response.

A non-targeting sgRNA control produced no significant changes in these response metrics (Supplementary Fig. 8b). The knockdown of TRPM7 also did not have any significant effect on the response of neurons to ultrasound $($ Fig. 5c). In contrast, the partial knockdown of TRPP1 and TRPP2 resulted in significant changes in the magnitude of the ultrasound response (Fig. 5, d-e). The CRISPR knockdown of Piezol did not result in a statistically significant change in the calcium signal, with the results showing a trend toward minor reduction (Fig. 5f). The knockdown TRPC1 resulted in a significant reduction in response $(\mathbf{F i g}$. 5g). Based on response reduction and CRIPSR knockdown efficiency for each channel, we can estimate their relative contributions to the ultrasound response of neurons, showing TRPP2 and TRPC1 
a

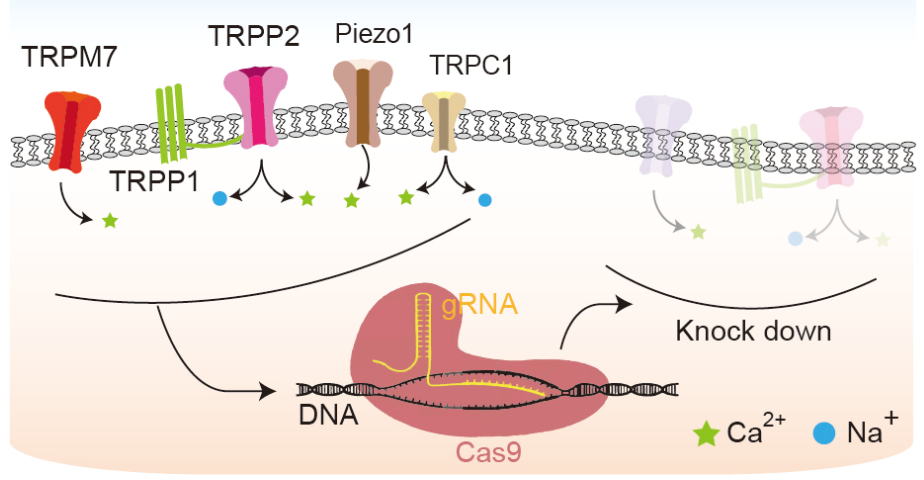

C

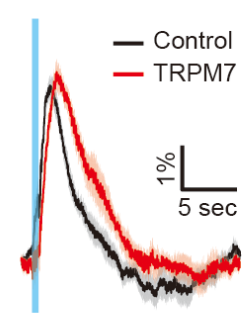

f

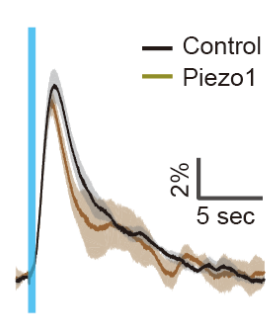

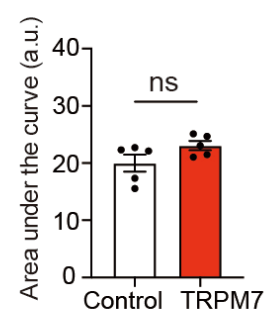

d
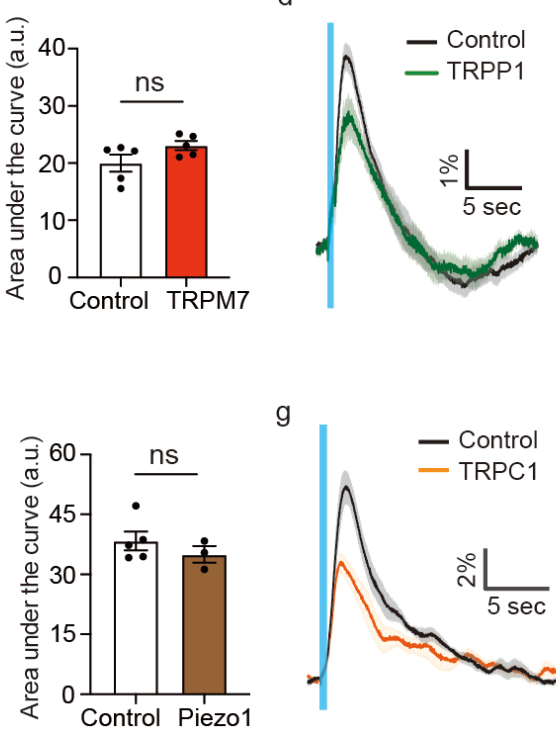

g

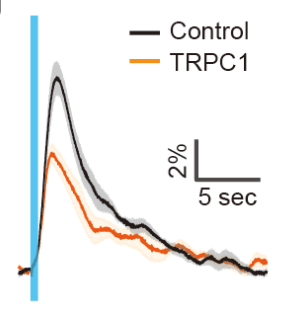

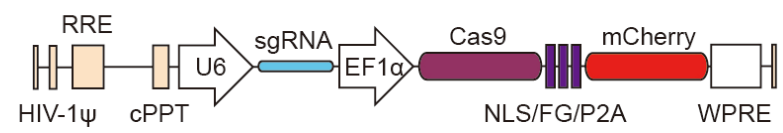
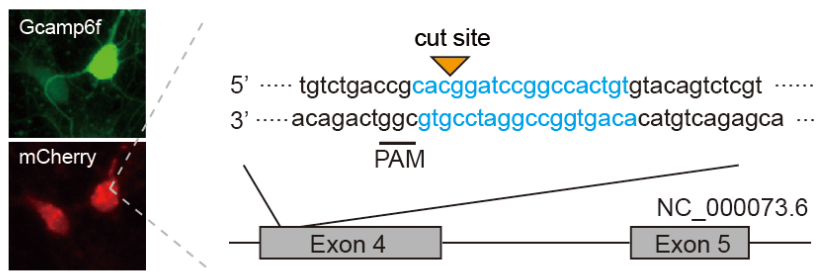

e
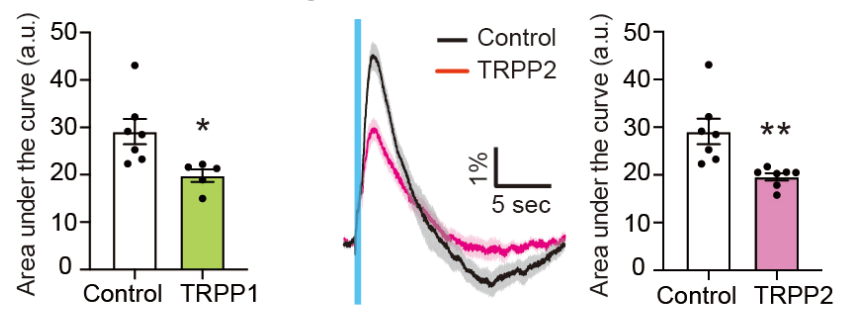

$\mathrm{h}$
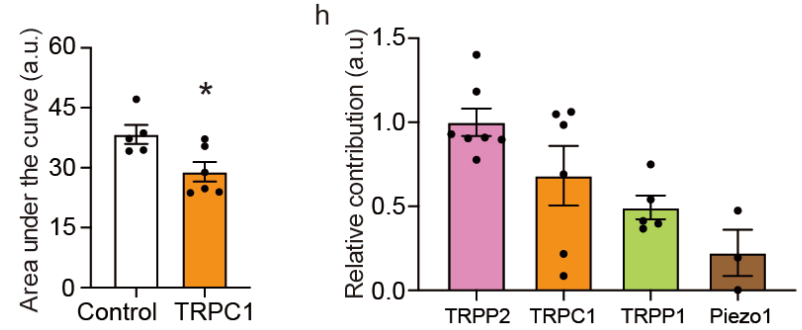

Fig.5 | GIRSPR/Cas9 knockdown of mechanosensitive ion channels. (a) Schematic of the strategy to knockdown individual ion channels using CRISPR/Cas9. (b) Schematic of the gene construct for the CRISPR knockdown. A sgRNA was designed to target each channel and delivered to neuron via lentivirus. (c) Calcium responses from wild-type neurons and neurons treated with CRISPR/Cas9 for TRPM7 knock down $(\mathrm{n}=5$ dishes each, Unpaired t test, $\mathrm{p}=0.1073)$. (d) Calcium responses from wild type neurons and modified neurons with CRISPR/Cas9 for TRPP1 knock down ( $\mathrm{n}=7$ dishes for control, and 5 dishes for TRPP1, Unpaired t test, $\mathrm{p}=0.0208)$. (e) Calcium responses from wild type neurons and modified neurons with CRISPR/Cas9 for TRPP2 knock down ( $\mathrm{n}=7$ dishes each, Unpaired $\mathrm{t}$ test, $\mathrm{p}=0.0084)$. (f) Calcium responses from wild type neurons and modified neurons with CRISPR/Cas9 for Piezol knock down ( $\mathrm{n}=5$ dishes for control, and 3 dishes for Piezol, Unpaired t test, $\mathrm{P}=0.3727$ ). (g) Calcium responses from wild type neurons and modified neurons with CRISPR/Cas9 for TRPG1 knock down ( $\mathrm{n}=5$ dishes for control, and 6 dishes for TRPC1, Unpaired t test, $\mathrm{p}=0.0232$ ). (h) Relative contribution of each channel to the ultrasound-evoked calcium response (normalized $\Delta \Delta \mathrm{F} / \mathrm{CRISPR}$ efficiency). Mean trace in solid and SEM is shaded in time courses.

to be the most important (Fig. 5h). No significant effects on baseline excitability were observed (Supplementary Fig. 8, c-d). Taken together, these results implicate the TRPP1/2 complex and TRPC1 mechanoreceptors in the neuronal response to ultrasound.

\section{Response to ultrasound is amplified by calcium-gated and low-threshold ion channels}

Since our calcium and voltage imaging experiments indicated that calcium entry was only the initial step in neuronal excitation, we endeavored to further examine the connection between mechanosensitive channel currents and the secondslong response of the neurons to ultrasound (Fig. 6a). In particular, we focused on the potential role of TRPM4, a non- selective cation channel expressed in cortical neurons, which is activated by intracellular $\mathrm{Ca}^{2+}$ at concentrations of $3 \mu \mathrm{M}$ and facilitates the amplification of small $\mathrm{Ca}^{2+}$ signals to larger depolarizing currents (Launay et al., 2002; Prawitt et al., 2003). We tested the involvement of TRPM4 in the ultrasound response by knocking it down with CRISPR/Cas9, as described above, with an efficiency of $43.4 \%$ (Supplementary Fig. 8a). Strikingly, we observed a major reduction in the response magnitude (Fig. 6b), strongly implicating this channel in the ultrasound response pathway. No effects on baseline excitability were observed due to this knockdown (Supplementary Fig. 8c).

Another set of potential downstream amplifiers are the voltagegated T-type calcium channels, which play an important role in triggering low-threshold spiking and action potential 


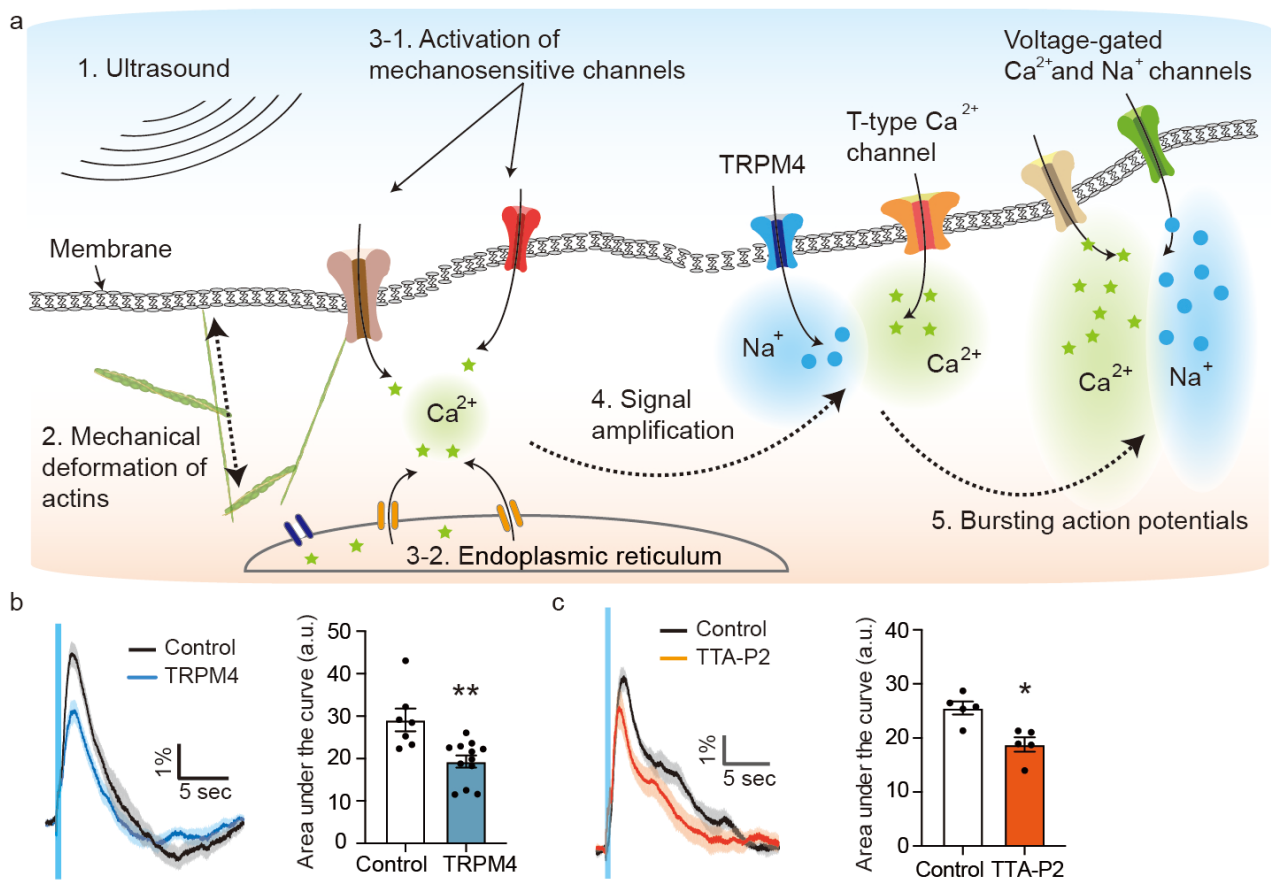

Fig.6 | Neuronal response to ultrasound is amplified by calcium-gated and voltagegated ion channels. (a) Illustration of the molecular pathway activated by ultrasound. (b) Calcium responses from wild type neurons and modified neurons with CRISPR/Cas9 for TRPM4 knock down $(\mathrm{n}=7$ dishes for control, and 12 dishes for TRPM4, Unpaired $\mathrm{t}$ test, $\mathrm{p}=0.0024)$. (c) Calcium responses before and after treatment with the t-type calcium channel blocker TTA-P2 $(3 \mu \mathrm{M}, \mathrm{n}=5$ dishes, paired T-test, $\mathrm{p}=0.0331$ ). Mean trace in solid and SEM is shaded.

bursting (Cain and Snutch, 2010). To test the involvement of this channel class, we treated the cells with the selective pore blocker TTA-P2 (Dreyfus et al., 2010) $\left(\mathrm{IC}_{50} \approx 100 \mathrm{nM}\right.$, used at $3 \mu \mathrm{M}$, Supplementary Fig. 7e). We observed a significant reduction in the amplitude of calcium responses $(\mathbf{F i g} . \mathbf{6 c})$. This result implicates the T-type calcium channels in generating the large and relatively long-lasting responses to ultrasound seen in our preparation and in animal studies (King et al., 2013; Xiaodan Niu, 2018; Yoo et al., 2011).

\section{Overexpression of mechanosensitive channels and amplifiers enhances the neuronal response to ultrasound}

To test our understanding of the molecular pathways underlying the ultrasound response and facilitate the development of sonogenetic strategies to sensitize genetically defined subsets of neurons to ultrasound, we overexpressed three of the ion channels identified in our knockdown experiments to have a role in this phenomenon. Based on their smaller size and ability to be packaged in lentiviral transfection vectors, we selected TRPC1, TRPP2 and TRPM4 as representative mechanoreceptors and calcium-dependent amplifier. Each gene was overexpressed in neurons under a hSyn promoter, as confirmed by immunofluorescent labeling (Fig. 7a). No or minor effects on baseline excitability were observed due to this overexpression (Supplementary Fig. 9, a-c). Strikingly, ultrasound stimulation of neurons overexpressing TRPC1 and TRPP2 elicited substantially larger calcium responses compared to wild-type cells, and enabled stronger activation at lower ultrasound intensities (Fig. 7, b-d).

Likewise, neurons overexpressing the TRPM4 channel showed marked increases their response amplitude (Fig. 7, b, e). These increases were significant under weak to high ultrasound intensity $\left(\geq 3 \mathrm{~W} / \mathrm{cm}^{2}\right)$. In addition, the overexpression of TRPM4 accelerated the kinetics of the ultrasound response, reducing the onset time of the calcium signal to below $100 \mathrm{msec}$ at $15 \mathrm{~W} / \mathrm{cm}^{2}$ (Fig. 7, h-j), consistent with previous overexpression studies (Launay et al., 2002). No significant changes in the onset kinetics were observed after overexpressing TRPG1 and TRPP2.

In contrast to these three channels, overexpression of TRPV1, which our inhibition experiments showed to be uninvolved in the neuronal response to ultrasound (Fig. 4d), produced no significant change in the neurons' ultrasound-elicited activation (Supplementary Fig. 9, d-e). Taken together, these results confirm the roles of TRPC1, TRPP2 and TRPM4 in the neuronal response to ultrasound and suggest that the overexpression of these channels can be used to sensitize neurons to this form of stimulation.

\section{DISCUSSION}

The results of this study provide a detailed biophysical and molecular description of the mechanisms by which ultrasound can excite neurons. Ultrasound has a mechanical interaction with the cell, causing the opening of specific calciumpermeable mechanosensitive ion channels, including TRPP1/2, TRPC1 and Piezo1. Calcium ions accumulate at a relatively low level over approximately $200 \mathrm{~ms}$ until they trigger the opening of calcium-sensitive sodium channels, including TRPM4. This leads to depolarization of the cell membrane and the opening of voltage-gated calcium channels, including T-type channels, leading to the large responses observable by GCaMP6f imaging (Fig. 6a).

The stimulation of neurons is repeatable, non-toxic and dosedependent on ultrasound intensity and pulse duration. Our data rule out temperature and synaptic neurotransmission as contributing mechanisms of ultrasonic neuromodulation. In addition, ultra-high frame rate imaging revealed no large-scale deformation or cavitation on the timescales of either the ultrasound cycle or the ultrasound pulse. Furthermore, no evidence was found to support a mechanism involving intramembrane cavitation and charge accumulation, as we did not observe the hyperpolarization predicted by the 
a

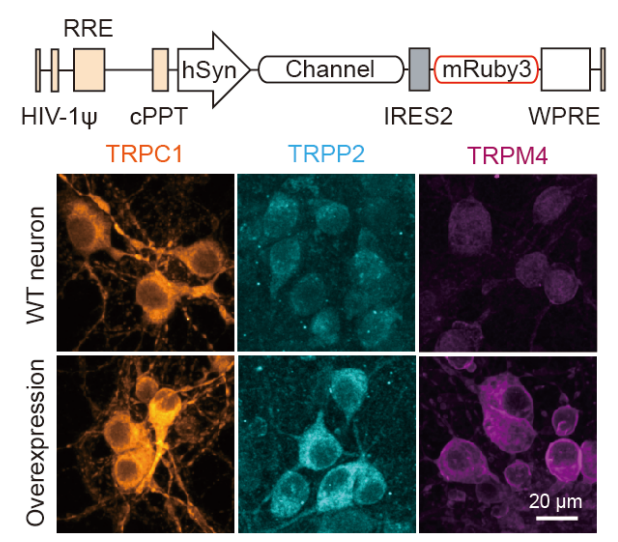

c

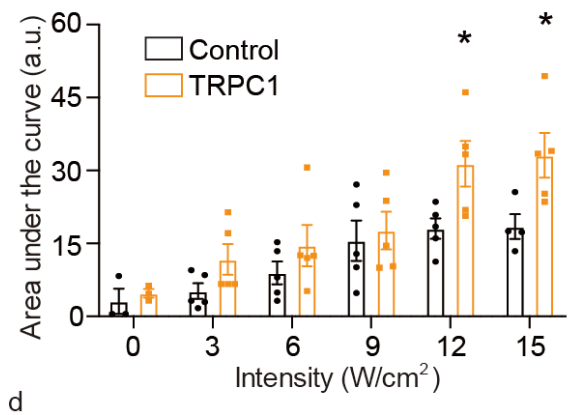

d

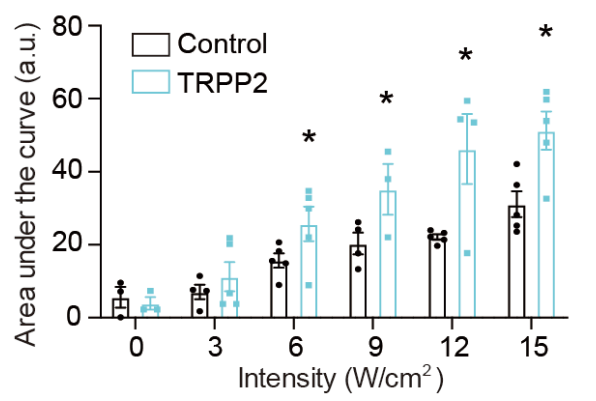

e b

0
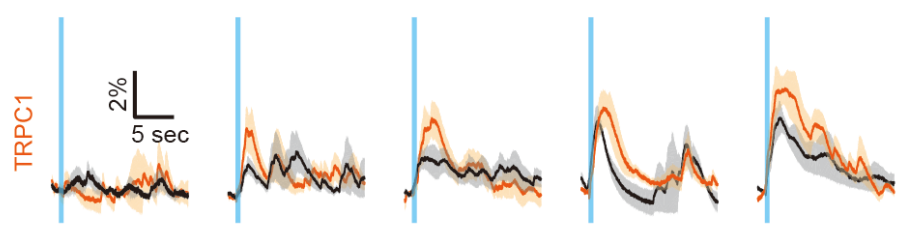

$15 \mathrm{~W} / \mathrm{cm}^{2}$
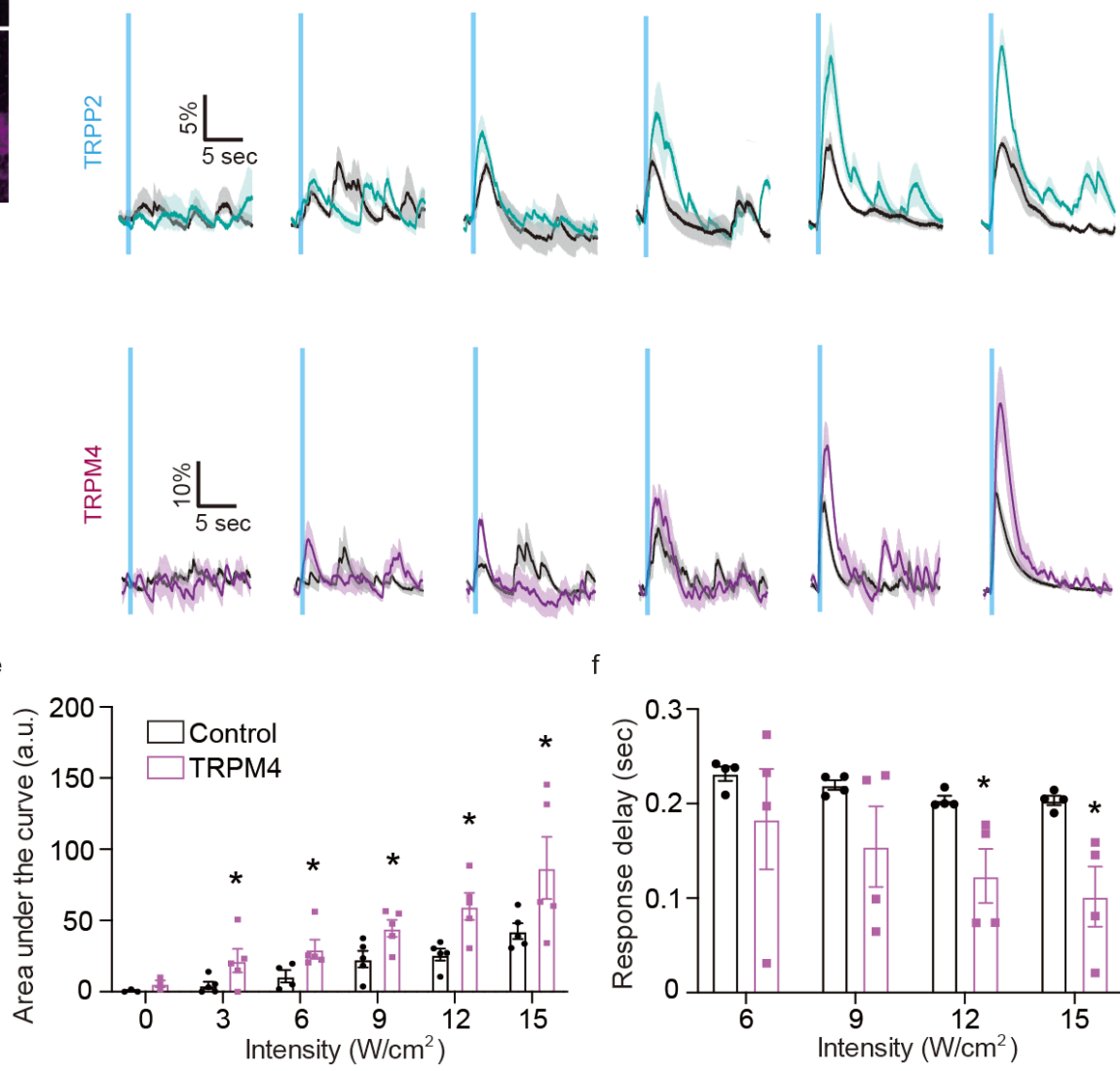

f

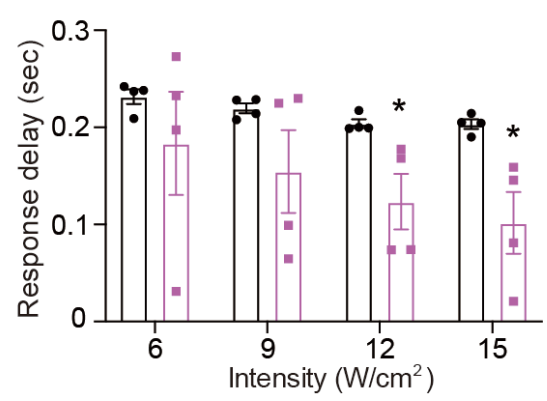

Fig.7 | Neuronal response to ultrasound is enhanced by overexpression of mechanosensitive and amplifier channels. (a) Schematic of genetic constructs for overexpressing TRPC1, TRPP2 and TRPM4 and immunostaining for the channels with and without overexpression. (b) Calcium responses from wild type neurons and overexpressing neurons as function of ultrasound intensity and quantification of area under the curve for (c) TRPG1 ( $\mathrm{n}=5$ dishes each, Unpaired T-test, $\left.\mathrm{p}=0.0316\left(12 \mathrm{~W} / \mathrm{cm}^{2}\right), \mathrm{p}=0.0369\left(15 \mathrm{~W} / \mathrm{cm}^{2}\right)\right),(\mathrm{d})$ TRPP2 $(\mathrm{n}=5$ dishes each, Unpaired T-test, $\left.\mathrm{p}=0.0855\left(6 \mathrm{~W} / \mathrm{cm}^{2}\right), \mathrm{p}=0.0795\left(9 \mathrm{~W} / \mathrm{cm}^{2}\right), \mathrm{p}=0.0105\left(12 \mathrm{~W} / \mathrm{cm}^{2}\right), \mathrm{p}=0.0127\left(15 \mathrm{~W} / \mathrm{cm}^{2}\right)\right)$, and $(\mathrm{e}) \mathrm{TRPM} 4(\mathrm{n}=$ 5 dishes each, Unpaired T-test, $\mathrm{p}=0.0815\left(3 \mathrm{~W} / \mathrm{cm}^{2}\right), \mathrm{p}=0.0578\left(6 \mathrm{~W} / \mathrm{cm}^{2}\right), \mathrm{p}=0.0317\left(9 \mathrm{~W} / \mathrm{cm}^{2}\right), \mathrm{p}=0.0114\left(12 \mathrm{~W} / \mathrm{cm}^{2}\right), \mathrm{p}=0.0841(15$ $\left.\mathrm{W} / \mathrm{cm}^{2}\right)$ ). (f) Comparison of response delay of calcium response between wild type and TRPM4-overexpressing neurons (Unpaired T-test, $\mathrm{p}=$ $\left.0.0321\left(12 \mathrm{~W} / \mathrm{cm}^{2}\right), \mathrm{p}=0.0442\left(15 \mathrm{~W} / \mathrm{cm}^{2}\right)\right)$. Mean trace in solid and SEM is shaded.

corresponding theory during the ultrasound pulse, did not detect any changes in the appearance of the neuronal membrane during ultra-fast imaging, and did not observe changes in neural responses after degassing the cell medium.

The use of primary cortical neurons as a model system allowed us to dissect the mechanisms of ultrasonic neuromodulation in comprehensive detail in the absence of the potential artifacts confounding in vivo studies, such as indirect auditory excitation (Guo et al., 2018; Sato et al., 2018). To ensure that our results are relevant for in vivo scenarios, we took care to culture neurons on an acoustically transparent substrate and confirmed that our ultrasound conditions elicited responses under both 2D and 3D culture conditions. Indeed, key features of the cultured neuron response to ultrasound matched those observed in viwo (King et al., 2013; Lee et al., 2018; Lee et al., 2016a; Lee et al., 2016b; Sharabi et al., 2018; Wattiez et al., 2017), including response latency and the range of responsive ultrasound intensities.

Several questions remain open for further study. Our experiments suggest, via the roles found for the actin cytoskeleton and mechanosensitive ion channels, that ultrasonic neuromodulation is mediated by mechanical stress on the plasma membrane. However, the precise forces and nanoscale deformations caused by ultrasound remain a subject for future research, which could include multiscale computational modeling and biophysical techniques 
specifically designed to measure nanoscale motion (Colom et al., 2018; Ha et al., 1996). These studies should further distinguish the roles of both traveling waves and static pressure gradients generated by beam focusing and reflections, which may be present in both in vivo and in vitro preparations (O'Reilly et al., 2010). In addition, while our study identified TRPP1/2, TRPC1 and Piezol as mechanosensitive ion channels involved the ultrasound response, the incomplete efficiency of our CRISPR knockouts makes it difficult for us to assess their relative roles. Alternative knockdown methods such as RNA interference or experiments with neurons derived from transgenic animals could provide further quantitative information. The relative expression of these channels in different neuronal subtypes may also impact the extent to which various populations of neurons in the brain respond to ultrasound.

The insights obtained in our study concerning the ion channels involved in ultrasonic neuromodulation may inform the development of sonogenetic strategies to sensitize specific brain regions and neuronal sub-populations to ultrasound (Ibsen et al., 2015; Maresca et al., 2018). Indeed, we showed that the overexpression of TRPC1, TRPP2 and TRPM4 increased the sensitivity of cortical neurons to ultrasound at reduced pulse intensities and durations, and in the case of TRPM4 greatly accelerated the response kinetics. Future work should focus on co-expressing these and other proteins identified in our study and applying them as sonogenetic agents in vivo.

In addition to neurons, it would also be interesting in future studies to examine the biophysical and molecular bases of ultrasonic stimulation in other cell types. For example, recent studies have demonstrated ultrasound-enhanced cholinergic signaling in the spleen (Gigliotti et al., 2013; Zachs et al., 2019), insulin release from pancreatic beta cells (Suarez Castellanos et al., 2017) and bone fracture healing (Zhang et al., 2012). Furthermore, overexpression of the mechanoreceptors and amplifier channels identified in this study could sensitize cells that do not have intrinsic ultrasound responses. We anticipate that the mechanistic insights obtained in this study will help stimulate each of these future research directions.

\section{MATERIALS AND METHODS}

\section{Primary neuron preparation}

All animal procedures were approved by the Institutional Animal Care and Use Committee of the California Institute of Technology. Gustom cell culture dishes were prepared from $3.5 \mathrm{~cm}$ diameter glass-bottom dishes (35pi, Matsunami, Osaka, Japan). The inner glass was removed by a diamond tip scribe (Fisher Scientific) and Mylar thin film (Chemplex, 2.5 $\mu \mathrm{m}$ thickness) was attached to the bottom of dish by polydimethylsiloxane (PDMS, sylgard 184, Dow), then baked for $3 \mathrm{~h}$ at $40^{\circ} \mathrm{C}$. Surfaces of the Mylar film were coated by polyD-lysine $(0.1 \mathrm{mg} / \mathrm{ml}$ in Trizma buffer, $\mathrm{pH} 8$, Sigma) overnight, and washed with deionized water followed by $70 \%$ ethanol and dried. Cortical tissues were dissected from embryonic day 18 C57BL/6J mice (The Jackson Laboratory). The tissues were rinsed with Hank's Balanced Salt Solution (VWR) and dissociated by pipetting, followed by centrifugation at 1000 rpm for $2 \mathrm{~min}$. Pellet was collected and re-suspended in culture medium. Cells were seeded on the top of Mylar dish at a density of 100 cells $/ \mathrm{mm}^{2}$ (for ultrasound stimulation experiments with minimum spontaneous activity) or 300 cells $/ \mathrm{mm}^{2}$ (for measuring spontaneous activity), and maintained in Neurobasal medium (Thermo Fisher Scientific) supplemented with B27 (2\% v/v, Thermo Fisher Scientific), GlutaMax (2mM, Gibco), glutamate (12.5 $\mu \mathrm{M}$, Sigma) and penicillin/streptomycin $(1 \% \mathrm{v} / \mathrm{v}$, Corning $)$ in a humidified incubator with $5 \% \mathrm{CO}_{2}$ and $37^{\circ} \mathrm{C}$. BrainPhys neuronal medium supplemented with SM1 (STEMCELL) was used in experiments involving the overexpression. Half of the medium was changed with the fresh medium without glutamate every 3 days, and neurons were used for ultrasound stimulation experiments after 12 14 days from the seeding.

For 3D neural tissue culture, Mylar dishes were pre-treated with oxygen plasma for $1 \mathrm{~min}$. Fibrillar collagen (Collagen I, Rat Tail, Gibco) was diluted to $2 \mathrm{mg} / \mathrm{ml}$ to mimic the stiffness of intact brain (Sharma et al., 2016). The center of the dish was filled with $200 \mu \mathrm{l}$ of the collagen mixture and incubated at room temperature for $30 \mathrm{~min}$ and washed with fresh medium. Re-suspended cells (50k cells) were mixed with $100 \mu \mathrm{l}$ of the collagen mixture and gently deposited onto the pre-gelled collagen, incubated for $30 \mathrm{~min}$ and washed with fresh medium. $1 \mathrm{ml}$ of the collagen mixture was then added to the dish and incubated for $1 \mathrm{~h}$ (for a total thickness $\sim 1 \mathrm{~mm}$ ). Then $1 \mathrm{ml}$ of fresh medium was filled after washing with the culture medium. For calcium imaging, Syn-driven GCaMP6f as a calcium sensor was delivered to neurons via AAV1 viral vector transfection (Addgene 100837-AAV1, 1E10 vp/dish) at 4 days in vitro. Membrane potential was optically imaged using an Ace2N voltage sensor (Gong et al., 2015). To construct the Ace2N-4AA-mNeon voltage sensor, the first 228 residues of the Acetabularia acetabulum rhodopsin II protein (GenBank: AEF12207) were codon-optimized for mouse cell expression and the cDNA was synthesized commercially (Integrated DNA Technologies). This was fused using a 5-residue linker (MLRSL) to the mNeonGreen protein (residues 14-236, GenBank: AGG56535), which was fused directly to a Golgi trafficking sequence (KSRITSEGEYIPLDQIDINV) and ER export tag (FCYENEV). The construct was cloned into a lentiviral transfer vector containing the woodchuck hepatitis virus posttranscriptional regulatory element (WPRE) (pLVX series, Clontech, Mountain View, CA) under the human synapsin 1 promoter (hSyn) with a strong Kozak sequence (GCCACG) using Gibson assembly. Lentiviral packaging was performed in HEK 293T cells using commercial plasmids (Addgene plasmids 12259 and 12263) and protocols. Lentivirus was applied to neurons at 3 days in vitro (1E9 $\mathrm{vp} /$ dish).

In preparation for voltage imaging and ultrasound stimulation under calcium-free conditions, the culture medium was replaced with artificial cerebrospinal fluid (ACSF) containing (in $\mathrm{mM}$ ) $25 \mathrm{NaHCO}_{3}, 10 \mathrm{D}$-glucose, $125 \mathrm{NaCl}, 2.5 \mathrm{KCl}, 1.25$ $\mathrm{NaH}_{2} \mathrm{PO}_{4}, 1 \mathrm{MgCl}_{2} 6 \mathrm{H}_{2} \mathrm{O}, 2 \mathrm{CaCl}_{2} 2 \mathrm{H}_{2} \mathrm{O}\left(0 \mathrm{CaCl}_{2} 2 \mathrm{H}_{2} \mathrm{O}\right.$ add 1 EGTA for calcium free ACSF) equilibrated with $95 \% \mathrm{O}_{2}$ and $5 \% \mathrm{CO}_{2}$. After the media replacement, cells were allowed to recover for $30 \mathrm{~min}$ in incubator.

For degassing the medium, $25 \mathrm{ml}$ of fresh medium in a $50 \mathrm{ml}$ tube was placed in a vacuum chamber to apply negative pressure (Welch, IL, -0.1 MPa). Boiling of the medium was 
seen in the first $5 \mathrm{~min}$, and additional degassing for $55 \mathrm{~min}$ was performed. After the degassing, normal culture medium was replaced with the degassed medium, and cell were allowed to recover for $30 \mathrm{~min}$ in incubator. The diffusion time of $\mathrm{O}_{2}$ or $\mathrm{CO}_{2}(>12 \mathrm{~h}$ for the $1 \mathrm{~cm}$ diffusion depth) (CUSSLER, 2009) was much slower than the total experiment time (45 min).

\section{Ultrasound stimulation setup and characterization of transducer}

A $300 \mathrm{kHz}$ ultrasound transducer (BII-7654/300IM, Benthowave Instrument INC. Canada) with $50 \mathrm{~mm}$ diameter and $24 \mathrm{~mm}$ focal distance was used in all experiments. The transducer was submerged in degassed water (degassed by a water conditioner, Onda, Aquas-10) and angled $20^{\circ}$ relative to normal incidence for the Mylar film using a customized holder. An Axon Digidata 1550 acquisition system (Molecular Devices, CA) was used to program and generate a set number of trigger pulses that were sent to an arbitrary waveform generator (Tabor Electronics, WX1282C) to generate the desired number of cycles of a sine wave at $300 \mathrm{kHz}$. The output of the generator was amplified by a linear amplifier $(75 \mathrm{~A} 250 \mathrm{~A}, \mathrm{RF}$ Microwave Instrumentation, PA) and used to drive the transducer. Calibration of the transducer and measurement of the pressure profile were done using a fiber optic hydrophone system (FOH, Precision Acoustics, UK) and optic hydrophones (PFS and TFS, Precision Acoustics, UK). The position of the hydrophone was controlled by stepping motor controllers (VELMEX INC., NY) while voltage traces were recorded by a digital oscilloscope (DSOX2004A, Keysight, CA) connected to a PC. From these measurements, the acoustic intensity of the ultrasound stimulus waveforms was calculated based on published standards (Tufail et al., 2010). To characterize the neuronal response to ultrasound at different acoustic intensities and durations, we randomized the sequence of the different waveforms to avoid accumulation effects.

\section{Fluorescence imaging of calcium and voltage}

A $490 \mathrm{~nm}$ LED light (LED4D067, Thorlabs, NJ) was used to excite the fluorescent proteins, and emitted signals were collected by an immersion lens (10x, NA 0.3, Leica) and recorded by a sCMOS camera (Zyla 5.5, Andor) at $100 \mathrm{~Hz}$ (200 $\mathrm{Hz}$ for voltage imaging). The recorded images were processed to extract calcium or voltage signals $(\mathrm{dF} / \mathrm{F})$ from each neuron by using NeuroCa (Jang and Nam, 2015). 50-300 cell bodies per each ROI (dish), depending on their seeding density, were detected by NeuroCa. Intensities during $500 \mathrm{~ms}$ before the onset of ultrasound stimulation were averaged, and this average was used as a baseline to calculate the area under the curve response to stimulation. Calcium signals within a time window between $0 \mathrm{sec}$ (onset of ultrasound) to $5 \mathrm{sec}$ were used for the calculation of area under the curve. To calculate calcium response delay, calcium signal was fitted using sigmoid fitting method (4-parameter logistic regression, Rsquare $>0.95)$, then $0.2 \%$ increase in the magnitude was set as onset time. To perform voltage imaging in a generic model of an excitable cell, we used spiking HEK cells (a gift from Adam E. Cohen) which were cultured as previously described (Park et al., 2013). We cloned an EFla-Ace2N-mNeon construct into a lentiviral transfer vector and performed lentiviral packaging using the protocol described above. Lentivirus (1E10 vp/dish) was applied to the spiking HEK cells at $50 \%$ confluency and centrifuged down onto the cells at $1500 \mathrm{~g}$ for 90 min with 10 $\mu \mathrm{g} / \mathrm{mL}$ polybrene. After 3 days of incubation at $37^{\circ} \mathrm{C}$, cells were treated with trypsin-EDTA $(0.25 \%$, Gibco $)$ for $1 \mathrm{~min}$ and plated on a mylar film dish at $80 \%$ confluency. After $24 \mathrm{~h}$ incubation at $37^{\circ} \mathrm{C}$, the cell medium was replaced with ACSF and incubated again for $30 \mathrm{~min}$ before voltage imaging. All data were analyzed using custom code written in MATLAB (Mathworks, MA). All values represent mean \pm SEM.

\section{Ultra-high-frame-rate optical imaging}

To observe cell membrane deformation at $\mathrm{MHz}$ frequencies we used a Shimadzu HPV-X2 camera. Samples were illuminated using a 2W $532 \mathrm{~nm}$ laser (CNI, MLL-F-532-2W) controlled by an optical beam shutter (Thorlabs SH05, KSC101). Right-angle prism mirrors directed the laser light through a water bath and into a sample dish containing the imaged neurons. The transducer was positioned in the water tank at an angle of $45^{\circ}$ relative to the water surface to minimize standing waves. $10 \mathrm{x}$ and $40 \mathrm{x}$ water immersion objectives (Leica, NA 0.3, Olympus, NA 0.8) were used. A series of prism mirrors and converging lenses with focal lengths of $200 \mathrm{~mm}$ and $50 \mathrm{~mm}$ delivered the image into the camera, which acquired 256 images over 51.2 us. Images were acquired starting $100 \mathrm{~ms}$ after the onset of ultrasound stimulation, to capture events coincident with the initiation of calcium and voltage signals. As a positive control for detecting large scale deformation, a PDL-coated mylar film dish was biotinylated by incubating NHS-biotin (Thermo Fisher Scientific, 2004 $\mu \mathrm{g} / \mathrm{ml}$ ) for $3 \mathrm{~h}$. After washing the free linkers with PBS, the dish was then incubated for $1 \mathrm{~h}$ with streptavidin-functionalized microbubbles (Advanced Microbubbles Laboratories LLC SIMB3-4SA, $4 \mu \mathrm{m}$ in diameter) to attach the bubbles onto the mylar film, and bubble cavitation was imaged using the same parameters as used with neurons.

\section{Cell viability and immunostaining}

Primary neurons were pre-treated with Calcein AM (Thermo Fisher Scientific), and live cells were imaged using $490 \mathrm{~nm}$ fluorescent excitation. Then, the neurons were stimulated with the highest intensity and longest duration ultrasound (15 $\mathrm{W} / \mathrm{cm}^{2}, 500 \mathrm{~ms}, 30$ times with 20 sec inter-pulse interval) and imaged again after a $1 \mathrm{~h}$ incubation. Live and dead cell counting was performed using Image $(\mathrm{NIH})$ to calculate the cell viability (Yoo and Nam, 2012). For immunostaining, primary neurons were fixed using ice-cooled paraformaldehyde (4\% in PBS, VWR) for $10 \mathrm{~min}$ at $4^{\circ} \mathrm{C}$, and washed with PBS. Nonspecific biding was blocked by $6 \%$ bovine serum albumin (Sigma) for $30 \mathrm{~min}$ at room temperature and cells were washed in PBS. Primary antibody (anti betatubulin (1:500, Sigma), Alexa Fluo 488 Phalloidin (1:500, Thermo Fisher Scientific), anti-TRPC1 (1:200, Alomone Labs), anti-TRPM4 (1:200, Alomone Labs) and anti-TRPP2 (1:200, Alomone Labs) were diluted in $1.5 \%$ bovine serum albumin, and incubated with cells for $1 \mathrm{~h}$. After washing with PBS, Hoechst 33342 (1:200, Sigma) was added to the PBS solution for nuclear staining. After 10 min, cells were washed with PBS, and imaged using a confocal microscope (LSM 880 with Airy scan, Zeiss). 


\section{Pharmacological treatments}

Chemicals blockers or peptide inhibitors (all from Tocris Bioscience, NM) applied directly in the media were used to block ion channels or manipulate cellular pathways. Identical volumes of buffer solutions were applied to control samples. A minimum concentration of TTX (final conc.: $1 \mu \mathrm{M}$ ) was titrated by monitoring the change of spontaneous calcium activity, and this was used to pharmacologically block voltagegated sodium channels. Thapsigargin (TG, final conc.: $500 \mathrm{nM}$ ) was used to block calcium pumps in the ER. Calcium release from ER after the TG application was confirmed by a transient calcium signal increase (Supplementary Fig. 3) (Wheeler et al., 2016). To block the presynaptic inputs, the postsynaptic blockers AP5 (final conc.: $1 \mu \mathrm{M}$ ) and CNQX (final conc.: $1 \mu \mathrm{M}$ ) (Yoo et al., 2014) were used. Actin filaments were depolymerized by their specific inhibitors, cytochalasin D (Cooper, 1987) and vinblastine, respectively(Moudi et al., 2013). Spontaneous calcium activities from separated groups were recorded before and after $1 \mathrm{~h}$ from the inhibitors treatment (final conc.: $1 \mu \mathrm{M}$ ), and the neurons were stimulated by ultrasound. After finishing the stimulation experiments, neurons were fixed for immunostaining. Minimum and working concentrations of chemical or peptide channel blockers were investigated by measuring the change of spontaneous calcium activities before and after applications (Supplementary Fig. 7). Gadolinium (Hamill and McBride, 1996) was applied to nonspecifically block the mechanosensitive ion channels (final conc.: $20 \mu \mathrm{M}$ ). After ultrasound stimulation, neurons were washed by fresh medium and incubated for $30 \mathrm{~min}$ for cell recovery, followed by ultrasound stimulation. Ruthenium red (final conc.: $1 \mu \mathrm{M}$ ) (Vriens et al., 2009) and TTA-P2 (final conc.: $3 \mu \mathrm{M}$ ) (Dreyfus et al., 2010) were treated before ultrasound stimulation to block TRP channels (TRPV1, 2, 4) and T-type calcium channels, respectively. To inhibit the GPCRs, suramin (Freissmuth et al., 1996) was added to medium (final conc.: $60 \mu \mathrm{M}$ ) and incubated with cells for $1 \mathrm{~h}$, then stimulated cells with ultrasound. GsMTx4 (Gnanasambandam et al., 2017) was added to medium (final conc.: $10 \mu \mathrm{M}$ ) and incubated with cells for $2 \mathrm{~h}$ to inhibit Piezol and TRPC1 channel gating, then stimulated cell with ultrasound.

\section{CRISPR/Cas9 for ion channel knockout}

3 sgRNAs for each target channel were designed using CRISPRko (Doench et al., 2016). Each sgRNA was inserted into a LentiCRISPR-mCherry backbone (Addgene, \#99154) and cloned by an established protocol (Ran et al., 2013). Lentivirus containing the sgRNA was delivered to neurons (1E9 vp/sample) at 3 days in vitro. After 10 days, genomic DNAs from the neurons was extracted using a DNA extraction kit (Qiagen), and CRISPR target regions were amplified by PCR. The PCR products were sequenced (Sanger sequencing), and the sequencing results were compared with those from wild-type neurons and non-targeting sgRNA to confirm the CRISPR knockout and to estimate knockout efficiency using the Tide tool (Brinkman et al., 2014). The most effective sgRNA was then choose among the 3 sgRNAs (Supplementary table 1) and its non-specific targeting efficiency was estimated by CFD score (Doench et al., 2016) (Supplementary table 2).

\section{Gene overexpression}

The mouse TRPV1 (GenBank: AB040873.1), TRPP2 (GenBank: BC053058) and TRPM4 (GenBank: BC096475), human TRPC1 (GenBank: Z73903.1), genes were synthesized commercially (Integrated DNA Technologies) and cloned upstream of an internal ribosome entry site (IRES2) and mScarlet (TRPC1, TRPP2) or mRuby3 (TRPV1, TRPM4) gene. The construct was inserted into the same lenti-backbone as described above. The viral particles were added to neurons at 3 days in vitro (1E9 vp/sample) and maintained for 10 days. To measure temperature change during ultrasound stimulation using mCherry, hSyn-driven mCherry was inserted into the lenti-backbone by Gibson assembly. The viral particles were added to neurons at 3 days in vitro (1E9 $\mathrm{vp} /$ sample), and maintained them for 10 days.

\section{ACKNOWLEDGEMENTS}

The authors thank Minjee Jang for assistance with calcium image processing and helpful discussions, and Tomokasu Sato for assistance with initial experiments, and all members of the Shapiro lab for helpful discussions and assistance with experiments. This research was supported by NIH BRAIN Initiative grants R24MH106107, RF1MH117080 (to M.G.S.) and NARSAD Young Investigator Grant (28802) from the Brain \& Behavior Research Foundation.

\section{AUTHOR CONTRIBUTIONS}

S.J.Y. and M.G.S. conceived this research. S.J.Y. and M.G.S. designed all experiments and S.J.Y. performed and analyzed the data. D.R.M. and S.J.Y. planned and performed the highspeed-imaging. R.H. and S.J.Y. planned and performed the gene construction. J.L. designed the initial optical setup for calcium imaging. S.J.Y. and M.G.S. wrote the manuscript with inputs from all authors.

\section{COMPETING FINANCIAL INTERESTS}

The authors declare no competing financial interests.

\section{DATA AND MATERIALS AVAILABILITY}

Raw data and genetic constructs are available upon request to the authors.

\section{MATERIALS AND CORRESPONDENCE}

Correspondence and material requests should be addressed to M.G.S. (mikhail@caltech.edu)

\section{REFERENCES}

Brinkman, E.K., Chen, T., Amendola, M., and van Steensel, B. (2014). Easy quantitative assessment of genome editing by sequence trace decomposition. Nucleic Acids Res 42, e168. 
Bystritsky, A., Korb, A.S., Douglas, P.K., Cohen, M.S., Melega, W.P., Mulgaonkar, A.P., DeSalles, A., Min, B.K., and Yoo, S.S. (2011). A review of low-intensity focused ultrasound pulsation. Brain Stimul 4, 125-136.

Cain, S.M., and Snutch, T.P. (2010). Contributions of T-type calcium channel isoforms to neuronal firing. Channels (Austin) 4, 475-482.

Chen, T.W., Wardill, T.J., Sun, Y., Pulver, S.R., Renninger, S.L., Baohan, A., Schreiter, E.R., Kerr, R.A., Orger, M.B., Jayaraman, V., et al. (2013). Ultrasensitive fluorescent proteins for imaging neuronal activity. Nature 499, 295-300.

Cheng, Y., Yao, H., Lin, H., Lu, J., Li, R., and Wang, K. (1999). The events relating to lanthanide ions enhanced permeability of human erythrocyte membrane: binding, conformational change, phase transition, perforation and ion transport. Chem Biol Interact 121, 267-289.

Christensen, A.P., and Corey, D.P. (2007). TRP channels in mechanosensation: direct or indirect activation? Nat Rev Neurosci $8,510-521$.

Colom, A., Derivery, E., Soleimanpour, S., Tomba, C., Molin, M.D., Sakai, N., Gonzalez-Gaitan, M., Matile, S., and Roux, A. (2018). A fluorescent membrane tension probe. Nat Chem 10, 1118-1125.

Constans, C., Mateo, P., Tanter, M., and Aubry, J.F. (2018). Potential impact of thermal effects during ultrasonic neurostimulation: retrospective numerical estimation of temperature elevation in seven rodent setups. Phys Med Biol 63, 025003. Cooper, J.A. (1987). Effects of cytochalasin and phalloidin on actin. J Cell Biol 105, 1473-1478.

GUSSLER, E.L. (2009). Values of Diffusion Coefficients. In Diffusion: Mass Transfer in Fluid Systems (Cambridge University Press).

Dallapiazza, R.F., Timbie, K.F., Holmberg, S., Gatesman, J., Lopes, M.B., Price, R.J., Miller, G.W., and Elias, W.J. (2018). Noninvasive neuromodulation and thalamic mapping with low-intensity focused ultrasound. J Neurosurg 128, 875-884.

Deffieux, T., Younan, Y., Wattiez, N., Tanter, M., Pouget, P., and Aubry, J.F. (2013). Low-intensity focused ultrasound modulates monkey visuomotor behavior. Curr Biol 23, 2430-2433.

Doench,J.G., Fusi, N., Sullender, M., Hegde, M., Vaimberg, E.W., Donovan, K.F., Smith, I., Tothova, Z., Wilen, C., Orchard, R., et al. (2016). Optimized sgRNA design to maximize activity and minimize off-target effects of CRISPR-Cas9. Nat Biotechnol 34, 184-191.

Dreyfus, F.M., Tscherter, A., Errington, A.C., Renger, J.J., Shin, H.S., Uebele, V.N., Crunelli, V., Lambert, R.C., and Leresche, N. (2010). Selective T-type calcium channel block in thalamic neurons reveals channel redundancy and physiological impact of I(T)window. J Neurosci 30, 99-109.

Folloni, D., Verhagen, L., Mars, R.B., Fouragnan, E., Constans, C., Aubry, J.F., Rushworth, M.F.S., and Sallet, J. (2019). Manipulation of Subcortical and Deep Cortical Activity in the Primate Brain Using Transcranial Focused Ultrasound Stimulation. Neuron 101, 1109-1116 e1105.

Freissmuth, M., Boehm, S., Beindl, W., Nickel, P., Ijzerman, A.P., Hohenegger, M., and Nanoff, C. (1996). Suramin analogues as subtype-selective G protein inhibitors. Mol Pharmacol 49, 602-611.

Gigliotti, J.C., Huang, L., Ye, H., Bajwa, A., Chattrabhuti, K., Lee, S., Klibanov, A.L., Kalantari, K., Rosin, D.L., and Okusa, M.D. (2013). Ultrasound prevents renal ischemia-reperfusion injury by stimulating the splenic cholinergic anti-inflammatory pathway. J Am Soc Nephrol 24, 1451-1460.

Gnanasambandam, R., Ghatak, C., Yasmann, A., Nishizawa, K., Sachs, F., Ladokhin, A.S., Sukharev, S.I., and Suchyna, T.M. (2017). GsMTx4: Mechanism of Inhibiting Mechanosensitive Ion Channels. Biophys J 112, 31-45.

Gong, Y., Huang, C., Li, J.Z., Grewe, B.F., Zhang, Y., Eismann, S., and Schnitzer, M.J. (2015). High-speed recording of neural spikes in awake mice and flies with a fluorescent voltage sensor. Science 350, 1361-1366.

Guo, H., Hamilton Ii, M., Offutt, S.J., Gloeckner, C.D., Li, T., Kim, Y., Legon, W., Alford, J.K., and Lim, H.H. (2018). Ultrasound Produces Extensive Brain Activation via a Cochlear Pathway. Neuron 99, 866.

Guo, M., Xu, Y., and Gruebele, M. (2012). Temperature dependence of protein folding kinetics in living cells. Proc Natl Acad Sci U S A 109, 17863-17867.

Ha, T., Enderle, T., Ogletree, D.F., Chemla, D.S., Selvin, P.R., and Weiss, S. (1996). Probing the interaction between two single molecules: fluorescence resonance energy transfer between a single donor and a single acceptor. Proc Natl Acad Sci U S A 93, 6264-6268.

Hamill, O.P., and McBride, D.W., Jr. (1996). The pharmacology of mechanogated membrane ion channels. Pharmacol Rev 48, 231-252.

Ibsen, S., Tong, A., Schutt, C., Esener, S., and Chalasani, S.H. (2015). Sonogenetics is a non-invasive approach to activating neurons in Caenorhabditis elegans. Nat Commun 6, 8264.

Jang, M.J., and Nam, Y. (2015). NeuroCa: integrated framework for systematic analysis of spatiotemporal neuronal activity patterns from large-scale optical recording data. Neurophotonics 2, 035003.

Kim, H., Taghados, S.J., Fischer, K., Maeng, L.S., Park, S., and Yoo, S.S. (2012). Noninvasive transcranial stimulation of rat abducens nerve by focused ultrasound. Ultrasound Med Biol 38, 1568-1575.

King, R.L., Brown, J.R., Newsome, W.T., and Pauly, K.B. (2013). Effective parameters for ultrasound-induced in vivo neurostimulation. Ultrasound Med Biol 39, 312-331.

Krasovitski, B., Frenkel, V., Shoham, S., and Kimmel, E. (2011). Intramembrane cavitation as a unifying mechanism for ultrasound-induced bioeffects. Proc Natl Acad Sci U S A 108, 3258-3263. 
Kubanek, J., Shukla, P., Das, A., Baccus, S.A., and Goodman, M.B. (2018). Ultrasound Elicits Behavioral Responses through Mechanical Effects on Neurons and Ion Channels in a Simple Nervous System. J Neurosci 38, 3081-3091.

Launay, P., Fleig, A., Perraud, A.L., Scharenberg, A.M., Penner, R., and Kinet, J.P. (2002). TRPM4 is a Ca2+-activated nonselective cation channel mediating cell membrane depolarization. Cell 109, 397-407.

Lee, W., Croce, P., Margolin, R.W., Cammalleri, A., Yoon, K., and Yoo, S.S. (2018). Transcranial focused ultrasound stimulation of motor cortical areas in freely-moving awake rats. BMC Neurosci 19, 57.

Lee, W., Kim, H., Jung, Y., Song, I.U., Chung, Y.A., and Yoo, S.S. (2015). Image-guided transcranial focused ultrasound stimulates human primary somatosensory cortex. Sci Rep 5, 8743.

Lee, W., Kim, H.C., Jung, Y., Chung, Y.A., Song, I.U., Lee, J.H., and Yoo, S.S. (2016a). Transcranial focused ultrasound stimulation of human primary visual cortex. Sci Rep 6, 34026.

Lee, W., Lee, S.D., Park, M.Y., Foley, L., Purcell-Estabrook, E., Kim, H., Fischer, K., Maeng, L.S., and Yoo, S.S. (2016b). Image-Guided Focused Ultrasound-Mediated Regional Brain Stimulation in Sheep. Ultrasound Med Biol 42, 459-470.

Legon, W., Ai, L., Bansal, P., and Mueller, J.K. (2018a). Neuromodulation with single-element transcranial focused ultrasound in human thalamus. Hum Brain Mapp 39, 1995-2006.

Legon, W., Bansal, P., Tyshynsky, R., Ai, L., and Mueller, J.K. (2018b). Transcranial focused ultrasound neuromodulation of the human primary motor cortex. Sci Rep 8, 10007.

Legon, W., Sato, T.F., Opitz, A., Mueller, J., Barbour, A., Williams, A., and Tyler, W.J. (2014). Transcranial focused ultrasound modulates the activity of primary somatosensory cortex in humans. Nat Neurosci 17, 322-329.

Leo Ai, P.B., Jerel K. Mueller and Wynn Legon (2018). Effects of transcranial focused ultrasound on human primary motor cortex using 7T fMRI: a pilot study. BMC Neuroscience 19.

Maresca, D., Lakshmanan, A., Abedi, M., Bar-Zion, A., Farhadi, A., Lu, G.J., Szablowski, J.O., Wu, D., Yoo, S., and Shapiro, M.G. (2018). Biomolecular Ultrasound and Sonogenetics. Annu Rev Chem Biomol Eng 9, 229-252.

Moudi, M., Go, R., Yien, G.Y., and Nazre, M. (2013). Vinca alkaloids. Int J Prev Med 4, 1231-1235.

Naor, O., Krupa, S., and Shoham, S. (2016). Ultrasonic neuromodulation. J Neural Eng 13, 031003.

Nightingale, K.R., Church, C.C., Harris, G., Wear, K.A., Bailey, M.R., Carson, P.L., Jiang, H., Sandstrom, K.L., Szabo, T.L., and Ziskin, M.G. (2015). Conditionally Increased Acoustic Pressures in Nonfetal Diagnostic Ultrasound Examinations Without Contrast Agents: A Preliminary Assessment. J Ultrasound Med 34, 1-41.

O'Brien, W.D., Jr. (2007). Ultrasound-biophysics mechanisms. Prog Biophys Mol Biol 93, 212-255.

O'Reilly, M.A., Huang, Y., and Hynynen, K. (2010). The impact of standing wave effects on transcranial focused ultrasound disruption of the blood-brain barrier in a rat model. Phys Med Biol 55, 5251-5267.

Oh, S.J., Lee, J.M., Kim, H.B., Lee, J., Han, S., Bae, J.Y., Hong, G.S., Koh, W., Kwon, J., Hwang, E.S., et al. (2020). Ultrasonic Neuromodulation via Astrocytic TRPAl. Curr Biol 30, 948.

Park, J., Werley, C.A., Venkatachalam, V., Kralj, J.M., Dib-Hajj, S.D., Waxman, S.G., and Cohen, A.E. (2013). Screening fluorescent voltage indicators with spontaneously spiking HEK cells. PLoS One 8, e85221.

Plaksin, M., Kimmel, E., and Shoham, S. (2016). Cell-Type-Selective Effects of Intramembrane Cavitation as a Unifying Theoretical Framework for Ultrasonic Neuromodulation. eNeuro 3.

Plaksin, M., Shoham, S., and Kimmel, E. (2014). Intramembrane Cavitation as a Predictive Bio-Piezoelectric Mechanism for Ultrasonic Brain Stimulation. Physical Review X 4, 011004.

Prawitt, D., Monteilh-Zoller, M.K., Brixel, L., Spangenberg, C., Zabel, B., Fleig, A., and Penner, R. (2003). TRPM5 is a transient Ca2+-activated cation channel responding to rapid changes in [Ca2+]i. Proc Natl Acad Sci U S A 100, 15166-15171.

Ran, F.A., Hsu, P.D., Wright, J., Agarwala, V., Scott, D.A., and Zhang, F. (2013). Genome engineering using the CRISPR-Cas9 system. Nat Protoc 8, 2281-2308.

Ranade, S.S., Syeda, R., and Patapoutian, A. (2015). Mechanically Activated Ion Channels. Neuron 87, 1162-1179.

Sanjana, N.E., Shalem, O., and Zhang, F. (2014). Improved vectors and genome-wide libraries for CRISPR screening. Nat Methods 11, 783-784.

Sato, T., Shapiro, M.G., and Tsao, D.Y. (2018). Ultrasonic Neuromodulation Causes Widespread Cortical Activation via an Indirect Auditory Mechanism. Neuron 98, 1031-1041 e1035.

Shapiro, M.G., Homma, K., Villarreal, S., Richter, C.P., and Bezanilla, F. (2012). Infrared light excites cells by changing their electrical capacitance. Nat Commun 3, 736.

Sharabi, S., Daniels, D., Last, D., Guez, D., Zivli, Z., Castel, D., Levy, Y., Volovick, A., Grinfeld, J., Rachmilevich, I., et al. (2018). Non-thermal focused ultrasound induced reversible reduction of essential tremor in a rat model. Brain Stimul.

Sharma, A., Licup, A.J., Rens, R., Vahabi, M., Jansen, K.A., Koenderink, G.H., and MacKintosh, F.G. (2016). Strain-driven criticality underlies nonlinear mechanics of fibrous networks. Phys Rev E 94, 042407.

Spassova, M.A., Hewavitharana, T., Xu, W., Soboloff, J., and Gill, D.L. (2006). A common mechanism underlies stretch activation and receptor activation of TRPC6 channels. Proc Natl Acad Sci U S A 103, 16586-16591.

Suarez Castellanos, I., Singh, T., Balteanu, B., Bhowmick, D.C., Jeremic, A., and Zderic, V. (2017). Calcium-dependent ultrasound stimulation of secretory events from pancreatic beta cells. J Ther Ultrasound 5, 30.

Tang, S.C., and Clement, G.T. (2010). Standing-wave suppression for transcranial ultrasound by random modulation. IEEE Trans Biomed Eng 57, 203-205. 
Tufail, Y., Matyushov, A., Baldwin, N., Tauchmann, M.L., Georges, J., Yoshihiro, A., Tillery, S.I., and Tyler, W.J. (2010). Transcranial pulsed ultrasound stimulates intact brain circuits. Neuron 66, 681-694.

Tyler, W.J. (2011). Noninvasive neuromodulation with ultrasound? A continuum mechanics hypothesis. Neuroscientist 17, 2536.

Tyler, W.J. (2012). The mechanobiology of brain function. Nat Rev Neurosci 13, 867-878.

Tyler, W.J., Tufail, Y., Finsterwald, M., Tauchmann, M.L., Olson, E.J., and Majestic, G. (2008). Remote excitation of neuronal circuits using low-intensity, low-frequency ultrasound. PLoS One 3, e3511.

Verhagen, L., Gallea, C., Folloni, D., Constans, C., Jensen, D.E., Ahnine, H., Roumazeilles, L., Santin, M., Ahmed, B., Lehericy, S., et al. (2019). Offline impact of transcranial focused ultrasound on cortical activation in primates. Elife 8.

Vriens, J., Appendino, G., and Nilius, B. (2009). Pharmacology of vanilloid transient receptor potential cation channels. Mol Pharmacol 75, 1262-1279.

Wagner, T., Valero-Gabre, A., and Pascual-Leone, A. (2007). Noninvasive human brain stimulation. Annu Rev Biomed Eng 9, 527-565.

Wang, G.J., Jackson, J.G., and Thayer, S.A. (2003). Altered distribution of mitochondria impairs calcium homeostasis in rat hippocampal neurons in culture. J Neurochem 87, 85-94.

Wattiez, N., Constans, C., Deffieux, T., Daye, P.M., Tanter, M., Aubry, J.F., and Pouget, P. (2017). Transcranial ultrasonic stimulation modulates single-neuron discharge in macaques performing an antisaccade task. Brain Stimul 10, 1024-1031.

Wheeler, M.A., Smith, G.J., Ottolini, M., Barker, B.S., Purohit, A.M., Grippo, R.M., Gaykema, R.P., Spano, A.J., Beenhakker, M.P., Kucenas, S., et al. (2016). Genetically targeted magnetic control of the nervous system. Nat Neurosci 19, 756-761.

Xiaodan Niu, K.Y., Bin He (2018). On the neuromodulatory pathways of the in vivo brain by means of transcranial focused ultrasound. Current Opinion in Biomedical Engineering 8.

Xu, J., Mathur, J., Vessieres, E., Hammack, S., Nonomura, K., Favre, J., Grimaud, L., Petrus, M., Francisco, A., Li, J., et al. (2018). GPR68 Senses Flow and Is Essential for Vascular Physiology. Cell 173, 762-775 e716.

Ye, P.P., Brown, J.R., and Pauly, K.B. (2016). Frequency Dependence of Ultrasound Neurostimulation in the Mouse Brain. Ultrasound Med Biol 42, 1512-1530.

Yoo, S., Hong, S., Choi, Y., Park, J.H., and Nam, Y. (2014). Photothermal inhibition of neural activity with near-infraredsensitive nanotransducers. ACS Nano 8, 8040-8049.

Yoo, S.J., and Nam, Y. (2012). Neurons on Parafilm: versatile elastic substrates for neuronal cell cultures. J Neurosci Methods 204, 28-34.

Yoo, S.S., Bystritsky, A., Lee, J.H., Zhang, Y., Fischer, K., Min, B.K., McDannold, N.J., Pascual-Leone, A., and Jolesz, F.A. (2011). Focused ultrasound modulates region-specific brain activity. Neuroimage 56, 1267-1275.

Younan, Y., Deffieux, T., Larrat, B., Fink, M., Tanter, M., and Aubry, J.F. (2013). Influence of the pressure field distribution in transcranial ultrasonic neurostimulation. Med Phys 40, 082902.

Zachs, D.P., Offutt, S.J., Graham, R.S., Kim, Y., Mueller, J., Auger, J.L., Schuldt, N.J., Kaiser, G.R.W., Heiller, A.P., Dutta, R., et al. (2019). Noninvasive ultrasound stimulation of the spleen to treat inflammatory arthritis. Nat Commun 10, 951.

Zhang, S., Cheng, J., and Qin, Y.X. (2012). Mechanobiological modulation of cytoskeleton and calcium influx in osteoblastic cells by short-term focused acoustic radiation force. PLoS One 7, e38343. 